\title{
Article
}

\section{HSP90 $\propto$ Mediates Sorafenib Resistance in Human Hepatocellular Carcinoma by Necroptosis Inhibition under Hypoxia}

\author{
Yan Liao ${ }^{1}$, Yue Yang ${ }^{1}$, Di Pan ${ }^{1}$, Youxiang Ding ${ }^{1}$, Heng Zhang ${ }^{1}$, Yuting $\mathrm{Ye}^{2}, \mathrm{Jia} \mathrm{Li}^{2}$ and Li Zhao ${ }^{1, *}$ \\ 1 School of Basic Medicine and Clinical Pharmacology, China Pharmaceutical University, Nanjing 211100, \\ China; 1831090212@stu.cpu.edu.cn (Y.L.); 1721091065@stu.cpu.edu.cn (Y.Y.); pdpharm@gmc.edu.cn (D.P.); \\ 1731090187@stu.cpu.edu.cn (Y.D.); 1821091168@stu.cpu.edu.cn (H.Z.) \\ 2 Pathology and PDX Efficacy Center, China Pharmaceutical University, Nanjing 211100, China;

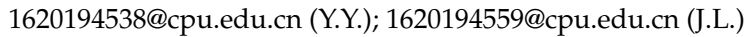 \\ * Correspondence: zhaoli@cpu.edu.cn
}

check for

updates

Citation: Liao, Y.; Yang, Y.; Pan, D.;

Ding, Y.; Zhang, H.; Ye, Y.; Li, J.; Zhao,

L. HSP90 $\propto$ Mediates Sorafenib

Resistance in Human Hepatocellular Carcinoma by Necroptosis Inhibition under Hypoxia. Cancers 2021, 13, 243. https://doi.org/10.3390/cancers 13020243

Received: 24 November 2020

Accepted: 4 January 2021

Published: 11 January 2021

Publisher's Note: MDPI stays neutral with regard to jurisdictional clai$\mathrm{ms}$ in published maps and institutional affiliations.

Copyright: (C) 2021 by the authors. Licensee MDPI, Basel, Switzerland. This article is an open access article distributed under the terms and conditions of the Creative Commons Attribution (CC BY) license (https:// creativecommons.org/licenses/by/ $4.0 /)$.
Simple Summary: Hypoxia is one of the characteristics of most solid tumors and induces cell resistant to chemotherapy. In this paper, we established a hypoxia model in both in vitro and in vivo to investigate the mechanisms of Sorafenib resistance in Hepatocellular carcinoma (HCC). Here, we observed that necroptosis could be an important target of Sorafenib in liver cancer and necroptosis blocking might be important in Sorafenib resistance under hypoxia. Mechanistically, our work suggests that HSP90 $\alpha$ plays a pivotal role in Sorafenib-induced necroptosis by binding with necrosome. HSP90 $\alpha$ could promote MLKL chaperone-mediated autophagy degradation in hypoxia, which subsequently decreased necroptosis. Consequently, the inhibition of necroptosis contributes to Sorafenib resistant. The Sorafenib resistance was reversed by HSP90 $\alpha$ inhibitorDemethoxygeldanamycin (17-AAG) in vivo and in vitro. This study highlights the important role of HSP90 $\alpha$ in Sorafenib resistance under hypoxia microenvironment, and provides a potential therapy target for liver cancer.

Abstract: As one of the most common malignancies worldwide, Hepatocellular carcinoma (HCC) has been treated by Sorafenib, which is the first approved target drug by FDA for advanced HCC. However, drug resistance is one of the obstacles to its application. As a typical characteristic of most solid tumors, hypoxia has become a key cause of resistance to chemotherapy and radiotherapy. It is important to elucidate the underlying mechanisms of Sorafenib resistance under hypoxia. In this study, the morphological changes of hepatocellular carcinoma cells were observed by Live Cell Imaging System and Transmission Electron Microscope; Sorafenib was found to induce necroptosis in liver cancer. Under hypoxia, the distribution of necroptosis related proteins was changed, which contributed to Sorafenib resistance. HSP90 $\alpha$ binds with the necrosome complex and promotes chaperone-mediated autophagy (CMA) degradation, which leads necroptosis blocking and results in Sorafenib resistance. The patient-derived tumor xenograft (PDX) model has been established to investigate the potential therapeutic strategies to overcome Sorafenib resistance. 17-AAG inhibited HSP90 $\alpha$ and presented obvious reversal effects of Sorafenib resistance in vivo and in vitro. All the results emphasized that HSP90 $\alpha$ plays a critical role in Sorafenib resistance under hypoxia and 17-AAG combined with Sorafenib is a promising therapy for hepatocellular carcinoma.

Keywords: hepatocellular carcinoma; necroptosis; hypoxia; HSP90 $\alpha$; sorafenib resistance

\section{Introduction}

Hepatocellular carcinoma (HCC) is reported to be the fifth most common cancer, with the second highest mortality among all cancers in adults [1]. At present, the com- 
mon treatment strategies for liver cancer are surgical resection, radiation therapies, and chemotherapy [2].

The multikinase inhibitor Sorafenib, originally developed as a Raf kinase inhibitor, targets not only the MAPK/ERK pathway, but also the vascular endothelial growth factor receptors (VEGF-R) and the platelet-derived growth factor receptor (PDGF-R) [3]. Sorafenib contributes to a survival benefit of patients through reducing tumor angiogenesis and increasing cancer cell apoptosis [4-6]. However, its efficacy has always been hampered by the occurrence of drug resistance [7-9], and HCC is much more difficult to cure after relapse. Therefore, the urgent problem to explore is the mechanism of Sorafenib resistance and to work out an effective treatment.

At present, it seems that necroptosis can be one of the important mechanisms of Sorafenib in the treatment of cancer [10-13]. Necroptosis is non-apoptotic cell death, which dependends on the receptor interacting protein kinase 3 (RIPK3). RIPK3 and RIPK1 can activate each other, promoting its conversion to an amyloid-like filamentous structure termed the necrosome, resulting in the recruitment of another necroptosis mediator, mixed lineage kinase domain-like (MLKL) [14,15]. Phosphorylated MLKL forms oligomers translocate to intracellular membranes and the plasma membrane, which eventually leads to membrane rupture. Recent evidence indicates that inhibition of caspase-dependent apoptosis sensitizes many cancer cells to necroptosis [11]. This has led to widespread interest in exploring necroptosis as an alternative strategy for anti-cancer therapy.

Hypoxia is one of the characteristics of most solid tumors, which plays an important role in the occurrence and development of cancers. Adaptation of tumor cells to hypoxia has important biological effects on drug resistance. Previous studies have reported that sustained Sorafenib treatment may promote hypoxia within tumors, which has been associated with Sorafenib resistance to HCC patients as well as subcutaneous mice model on HCC [16]. Hypoxia usually results in the resistance of various tumors to therapy through inducing the activation of the HIF signaling pathway and the survival of tumor cells $[17,18]$. Molecular chaperones are a heterogeneous class of proteins unified by their primary function of assisting the cellular proteome to achieve and maintain a conformationally mature and functional state $[19,20], \mathrm{HSP} 90 \alpha$ is a chaperone protein that interacts with client proteins that it is closely related to cell apoptosis, metastasis, invasion and chemotherapy resistance, it protects cells from damage and stimuli, promotes tumor cells growth, make tumor cells tolerate chemotherapy, heat treatment and other traumatic stimuli, and finally leads to treatment failure. In HCC, HSP90 $\alpha$ expression positively correlated with HIF-1. Down-regulation of HIF- $1 \alpha$ or HIF-1 $\beta$ completely blocks HSP90 $\alpha$ secretion, indicating that HIF-1 is a critical upstream regulator of HSP90 $\alpha$ secretion [21]. In recent years, HSP90 $\alpha$ also proved to play an important role in drug resistance under hypoxia [22-25]. Demethoxygeldanamycin (17-AAG) is a derivative of geldanamycin that is currently undergoing clinical development as a novel anticancer agent for the treatment of human cancers [26,27]. 17-AAG induces tumor apoptosis and inhibits tumor proliferation in leukemia cells and prostate cancer has already been studied. The combination of 17AAG and oxaliplatin or capecitabine in colorectal cancer cell lines has been studied. 17AAG in combination with paclitaxel on anaplastic thyroid carcinoma cells has also been reported [28-30]. In this study, we confirmed that necroptosis was one of the important reasons why Sorafenib attacks HCC. Besides, we elucidated that $\mathrm{HSP} 90 \alpha$ binds with the RIPK1/RIPK3/MLKL complex to promote chaperone-mediated autophagy (CMA) degradation, which would be the main cause of Sorafenib resistance. 17-AAG, as a specific inhibitor of HSP90 $\alpha$, could overcome Sorafenib resistance on HCC. Combining 17-AAG with Sorafenib might be a potential therapeutic strategy to enhance Sorafenib efficacy for the treatment of HCC. 


\section{Results}

\subsection{Sorafenib Induced HCC Necroptosis}

Soft agar assay (Figure 1A) and Flow cytometry (Figure 1B) were used to detect the inhibitory effect of Sorafenib on HCC cell lines. Results suggested that, after being treated with $10 \mu \mathrm{M}$ Sorafenib for $24 \mathrm{~h}$, HepG2 and Huh7 cells growth was decreased to $9.5 \%$ and $16 \%$, compared to the control group, respectively. The dead cells were increased to $35.1 \%$ and $18.1 \%$, respectively.

Figure 1C,D showed the morphological changes in HepG2 cells by Live Cell Imaging System and a Transmission Electron Microscope. We can see that cells experienced normal mitotic proliferation in the control group. While in the Sorafenib treated group, cells suffered a blocked proliferation, cellular content aggregates, and eventually swelled until dead (Figure 1C). More accurate views of various changes in cell death patterns were investigated by a Transmission Electron Microscope. As shown in Figure 1D, the healthy state of liver cancer cells was presented in the control group. Picture No. 1 showed the complete organelles like mitochondria and it has complete cell membrane structures. However, Sorafenib induced cell death, including necroptosis, apoptosis, and autophagic death, etc. In Picture No. 2, cell membrane and organelles that were destroyed are shown in the Sorafenib treated group. Cells swelled, cell content was released, and the morphology of the main cell main presented as necrosis. We also observed an apoptotic body in Picture No. 3 and autophagosomes in Picture No. 4. In Figure 1E,F, the expression of necroptosis marker proteins such as RIPK1, RIPK3, and MLKL were detected by western blot and immunofluorescence. The results showed that RIPK1, RIPK3, and MLKL were increased in treated cells, which strongly suggested that Sorafenib activated the necroptosis pathway. Additionally, the necroptosis marker proteins were detected in tumor tissues in the PDX model. WB (Figure 1G) and IHC (Figure 1H) results showed that Sorafenib induced necroptosis in vivo as well. Besides, the HepG2 cells xenograft model was used to confirm the results (Figure S3A,B). All the results indicated that Sorafenib induced HCC necroptosis in vitro and in vivo.

Apoptotic cell death involves the engagement of pathways that result in the activation of caspase proteases that ultimately cause the morphological features of cell death. In contrast, necroptosis was recognized as a caspase-independent cell death that can be triggered by tumor necrosis factor (TNF) in the presence of a pan-caspase inhibitor such as zVADfluoromethylketone (VAD). To detect the necroptosis inducing effect of Sorafenib, Nec, a specific inhibitor of RIPK1 kinase was used. As shown in Figure 1I, Sorafenib induced obvious cell death even when VAD existed, and the effect was reversed by Nec, which suggested that the necroptosis inducing effect of Sorafenib on HCC cells was apoptosis independent. The morphological changes of HepG2 cells under Sorafenib were observed by Live Cell Imaging System (Figure 1J). Transmission Electron Microscope showed similar results (Figure S3C). In the HepG2 xenograft model, Sorafenib inhibited tumor growth significantly, while Nec weakened the effect (Figure S3D,E). All the results showed that Sorafenib induced hepatocellular carcinoma death partly via the necroptosis pathway in vivo and in vitro. 
A
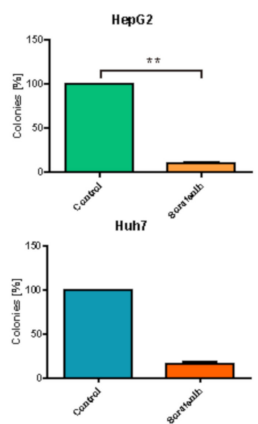

B
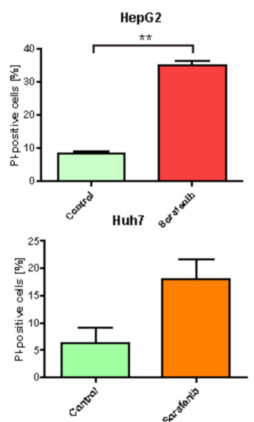

$\mathrm{D}$
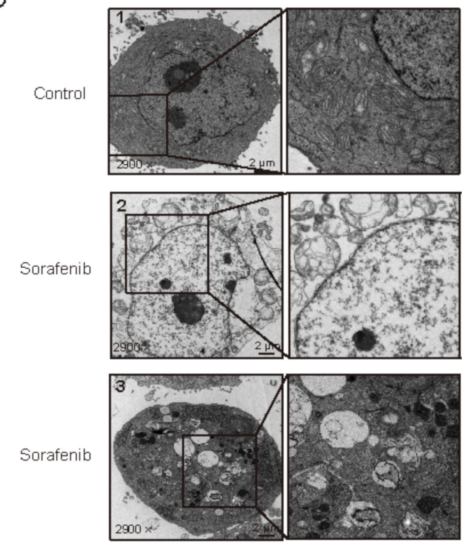

C
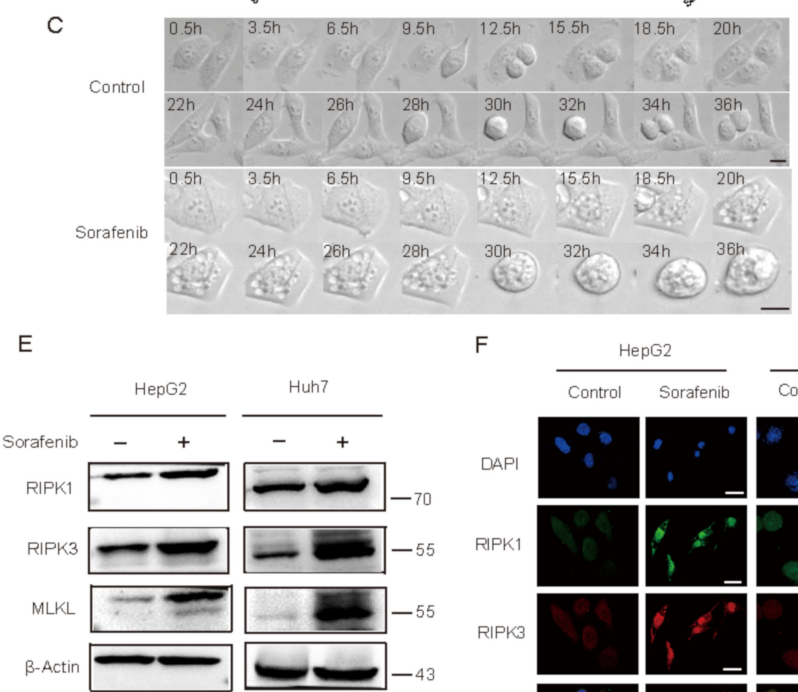

$\mathrm{F}$

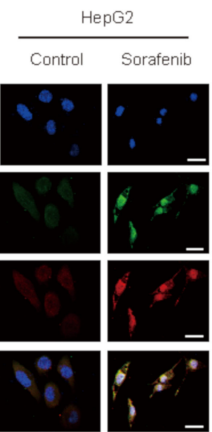

J
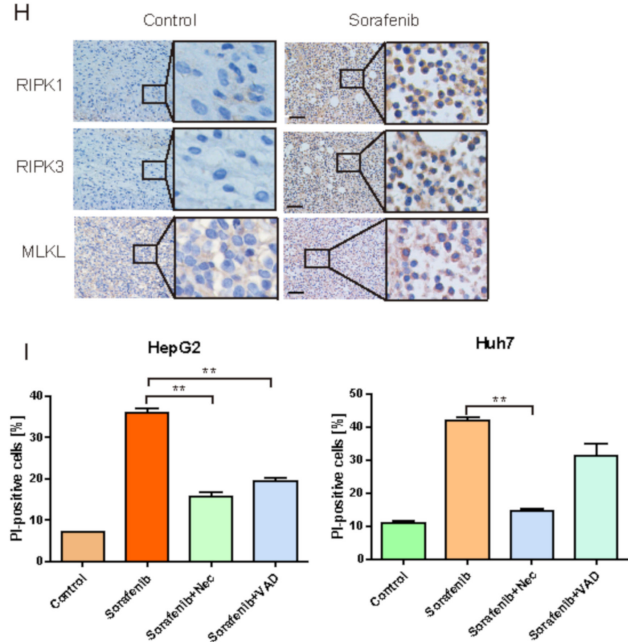
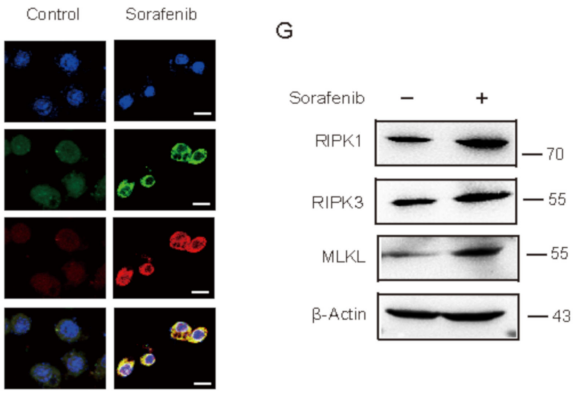

$G$
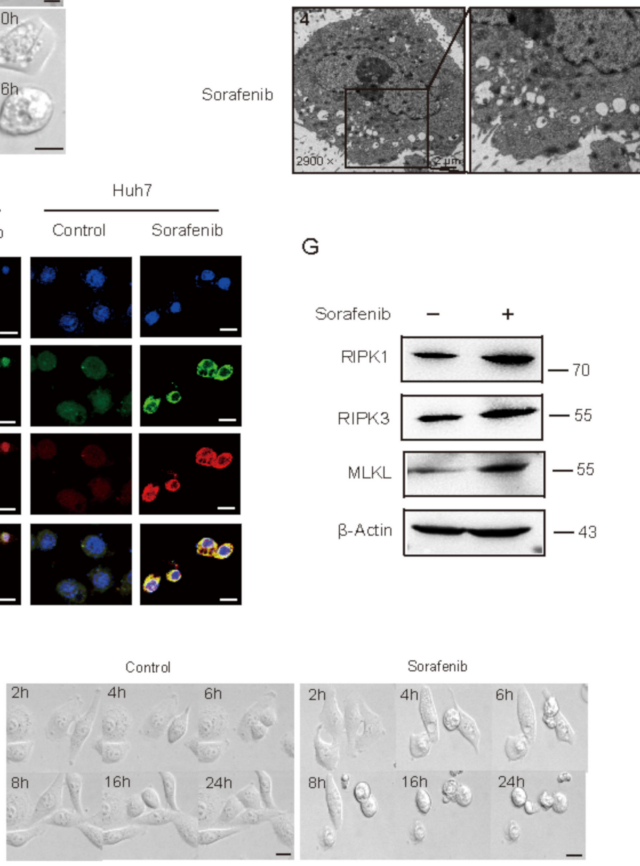

Sorafenib+Nec

Sorafenib+VAD

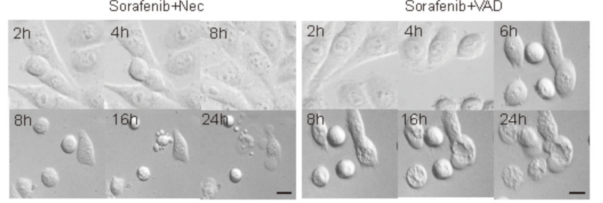

Figure 1. Sorafenib Induced HCC Necroptosis. (A) The inhibitory effect of Sorafenib on Hepatocellular carcinoma cell lines by Soft Agar Assay. The results were shown as mean \pm SEM from three independent experiments. ${ }^{* *} p<0.01$. (B) Detection of PI-positive cells in Huh7 and HepG2 cells with Sorafenib treatment by Flow Cytometry. The results were shown as mean \pm SEM from three independent experiments. ${ }^{* *} p<0.01$. (C) To observed the morphological changes of HepG2 cells by Live Cell Imaging System. Scale bar $=40 \mu \mathrm{m}$. (D) The different types of death in HepG2 cells induced by Sorafenib observed by Transmission Electron Microscope. (E,F) The expressions of necroptosis pathway in hepatocellular carcinoma cell lines after Sorafenib treated detected by Western blot and Immunofluorescence. Scale bar $=40 \mu \mathrm{m}$. (G,H) The expression of RIPK1, RIPK3 and MLKL were dectected in PDX-xenograft respectively by Western blot and IHC. Scale bar $=800 \mu \mathrm{m}$. (I) Detection of PI positive cells by flow cytometer to observe the dead cell when Nec and VAD exists. The results were shown as mean \pm SEM from three independent experiments. ${ }^{* *} p<0.01$. (J) Live cell Imaging System observed morphological changes in HepG2 cells when Nec and VAD exist. Scale bar $=40 \mu \mathrm{m}$. 


\subsection{Hypoxia Contributed to HCC Resistance to Sorafenib}

Hypoxia can promote proliferation, invasion, metastasis, apoptosis, drug resistance and other malignant biological behaviors of tumor cells. The IC50 values of Sorafenib under normoxia and hypoxia were tested (Figure 2A). In HepG2 cells, the IC50 was $12.8 \mu \mathrm{M}$ under normoxia and $194.2 \mu \mathrm{M}$ under hypoxia. In Huh7 cells, the values were $11.1 \mu \mathrm{M}$ and $34.7 \mu \mathrm{M}$. Clone formation experiment also confirmed that hypoxia can induce Sorafenib resistant under hypoxia (Figure 2B). Further, the results of trypan blue staining also showed that cell death induced by Sorafenib was significantly reduced under the hypoxia condition (Figure S3F). WB results proved that the necroptosis pathway induced by Sorafenib was attenuated under hypoxia (Figure 2C). The results of Co-IP also revealed that the capacity of RIPK1 binding with RIPK3/MLKL was upregulated in normoxia and decreased in the hypoxia microenvironment after Sorafenib treatment, which indicated that hypoxia reduced the activation necroptosis pathway of Sorafenib (Figure 2D).

A
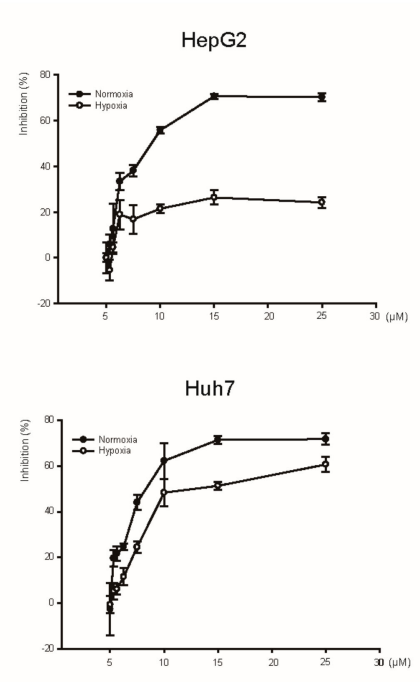

B

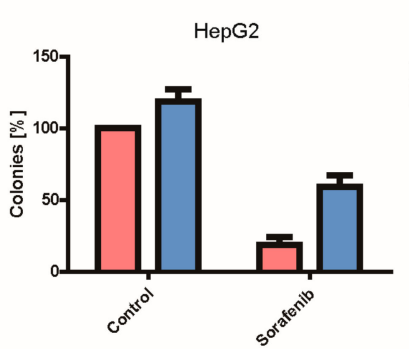

Huh7

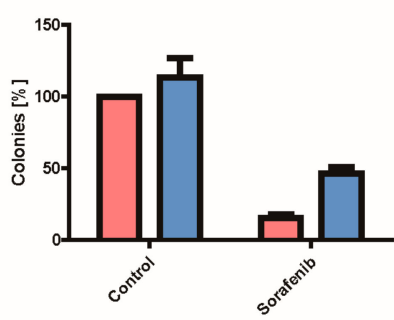

C

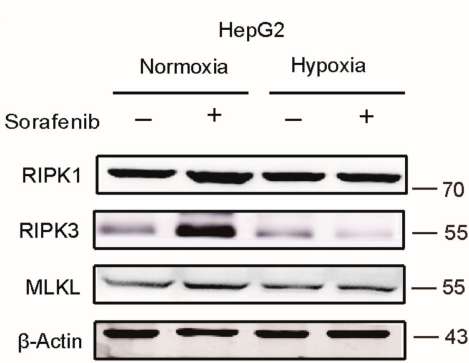

므 Nypoxia

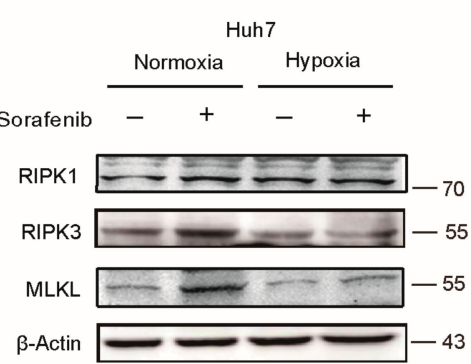

D

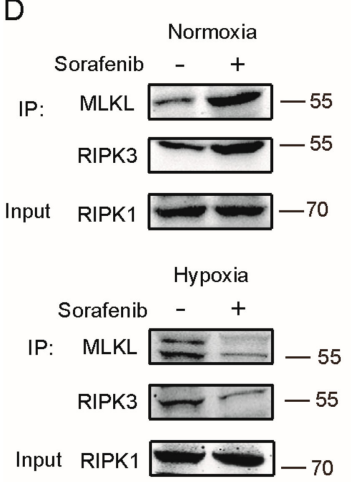

$E$

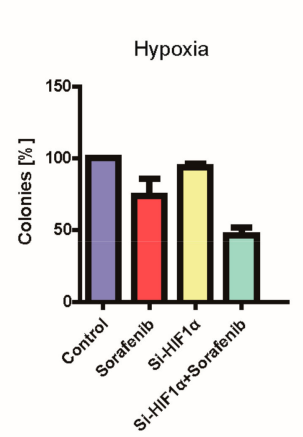

$\mathrm{F}$

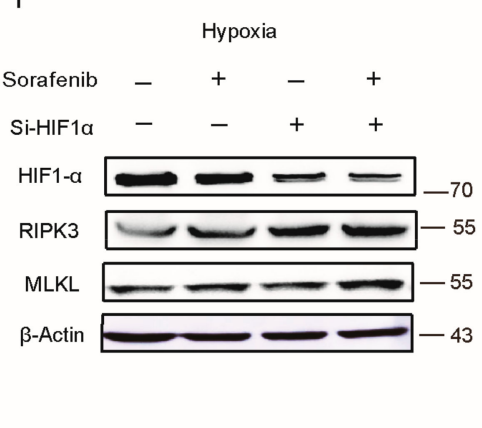

G

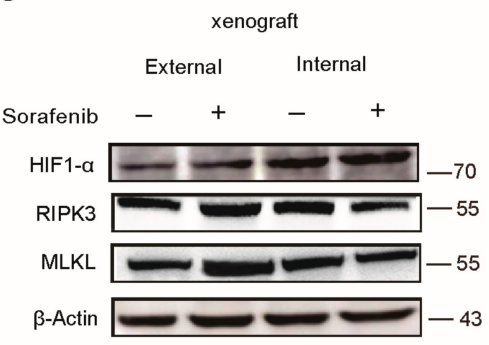

Figure 2. Hypoxia Induced Hepatocellular Carcinoma Resistant to Sorafenib. (A) Inhibition of Sorafenib under normoxia and hypoxia assessed by MTT assay. (B) Inhibition of Sorafenib under normoxia and hypoxia assessed by Clone formation experiment. (C) The expression of necroptosis proteins in normoxia and hypoxia detected by Western Blot. (D) The binding capacity of the necroptosis complex under normoxia and hypoxia. (E,F) HIF1 $\alpha$ effect on Sorafenib observed by Soft Agar Cloning experiment. (F) HIF1 $\alpha$ effect on Sorafenib in necroptosis pathway detected WB. (G) Detected HIF1 $\alpha$ and necroptosis protein in PDX-xenograft tumor tissues by WB.

HIF $1 \alpha$ (hypoxia inducible factor-1) is a key regulator of many signals in tumor occurrence, development, and chemotherapy resistance. In order to figure out the effect of HIF1 $\alpha$ on Sorafenib resistance, HIF1- $\alpha$ siRNA was used. Soft Agar Cloning experiment 
showed that HIF1 $\alpha$ knockdown can enhance the inhibitory effect of Sorafenib (Figure 2E). Results in Figure 2F showed that RIPK3 and MLKL in HepG2 cells were upregulated by Sorafenib with HIF1 $\alpha$ being silenced under hypoxia. In the PDX model (Figure 2G), HIF1 $\alpha$ was much more expressed in internal hypoxia tumor tissue than in external normoxia tumor. Additionally, Sorafenib could not activate the necroptosis pathway in the tumor internal area, which means hypoxia blocked the necroptosis induced by Sorafenib in vivo.

\subsection{Hypoxia Impeded the Distribution of RIPK1/RIPK3/MLKL Complex in Cytoplasm}

The necroptosis key protein MLKL usually acts on lipid and cell membrane structures, causing membrane damage and eventually leading to cell death [31,32]. In order to figure out the mechanisms of Sorafenib resistance under hypoxia, the changes in MLKL expression and the complex of RIPK1/RIPK3/MLKL in cytoplasm were studied. In Figure 3A, under normoxia, RIPK1, RIPK3, and MLKL were increased by Sorafenib in cytoplasm. However, under hypoxia, necroptosis related proteins were down-regulated in cytoplasm. The RIPK1/RIPK3/MLKL complex was also detected by Co-IP (Figure 3B). Results showed that, in cytoplasma, Sorafenib increased the RIPK1/RIPK3/MLKL complex under normoxia and had no obvious effect under hypoxia. Therefore, Sorafenib could not increase necrosome in cytoplasm, which might be an important reason for Sorafenib resistance under hypoxia.

In this study, Sorafenib-induced necroptosis destroy the membrane structures in hepatocellular carcinoma cells. Sequently we detected the distribution of MLKL in several kinds of organelles intracellular which has abundant membranous structure, such as cell membrane, mitochondria, endoplasmic reticulum, lysosomes. As shown in Figure 3C-F, MLKL increased after Sorafenib treatment in normoxia and was located in various organelles. However, the location was decreased in organelles and increased in the nucleus under hypoxia. Similar results were observed in primary tumor cells (Figure S2A-D). All the results indicated that MLKL was strongly located in the kinds of organelles that had membranous structures in the process of Sorafenib induced necroptosis; then, the damaged membranes finally lead to cell death. Hypoxia reduced MLKL expression in organelles, which might be one of the main reasons why necroptosis induced by Sorafenib was attenuated under hypoxia.

\subsection{HSP90 $\alpha$ Promotes Chaperone-Mediated Autophagy (CMA) Degradation by Directly Binding to MLKL in Hypoxia}

To investigate whether hypoxia could affect the necroptosis protein degradation under Sorafenib treatment, protein synthesis inhibitor CHX was performed. As shown in Figure 4A, MLKL did not change when CHX was used in normoxia. While under hypoxia, MLKL was time-dependently decreased by Sorafenib. It seemed that MLKL was more susceptible to degrade under hypoxia than under nomoxia when cells were co-cultured with Sorafenib. Generally, there are three main ways that proteins are degraded: the proteasome pathway, caspase pathway, and autophagy-lysosome pathway. To verify which pathway contributed to the MLKL degradation under hypoxia, proteasome inhibitor MG132, caspase inhibitor z-VAD-fmk, and autophagy inhibitor chloroquine were used. As shown in Figure 4A and Figure S2E, rather than MG132 or z-VAD-fmk, the chloroquine abrogated the degradation of MLKL, indicating that the autophagy-lysosome pathway was the main degradation of MLKL in the hypoxia microenvironment. 
A

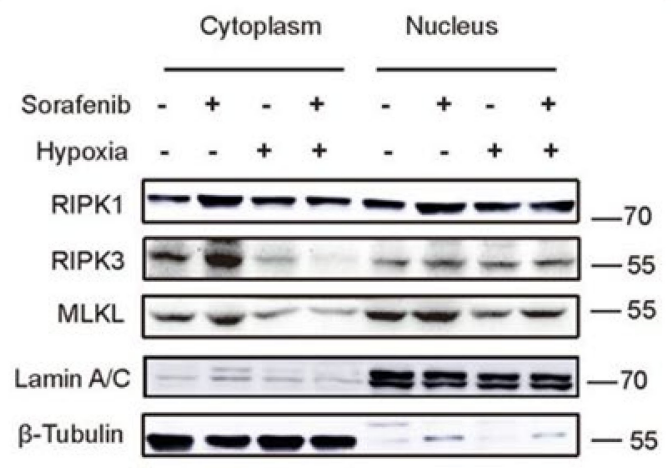

B

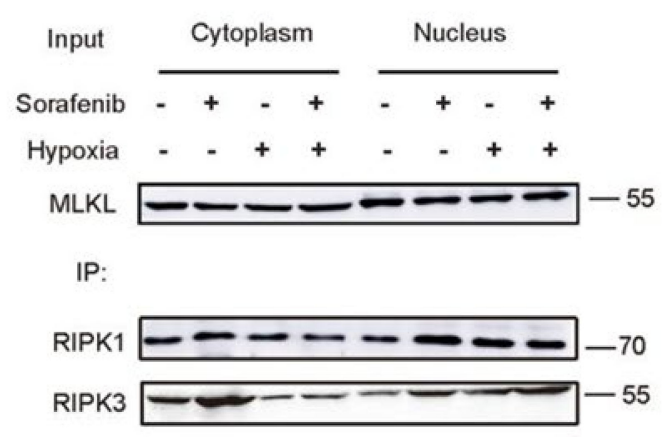

C

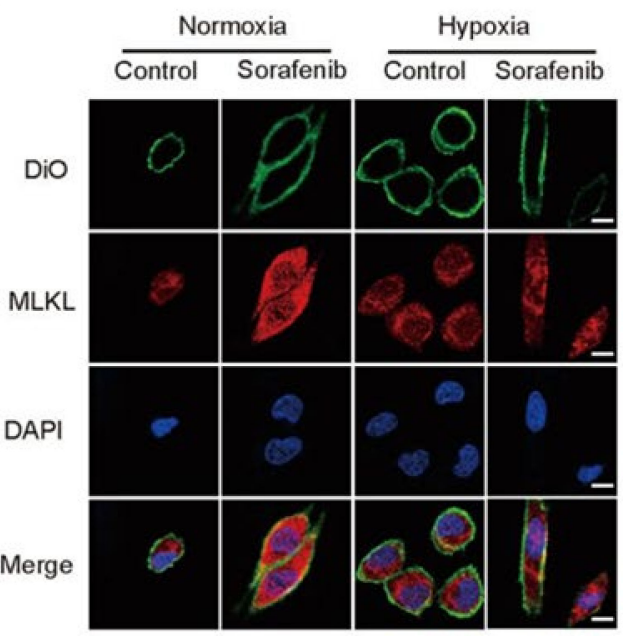

D

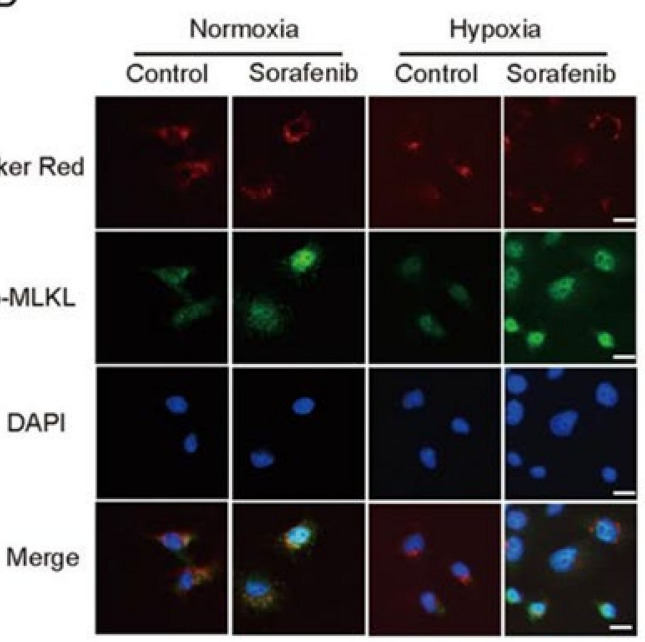

$E$

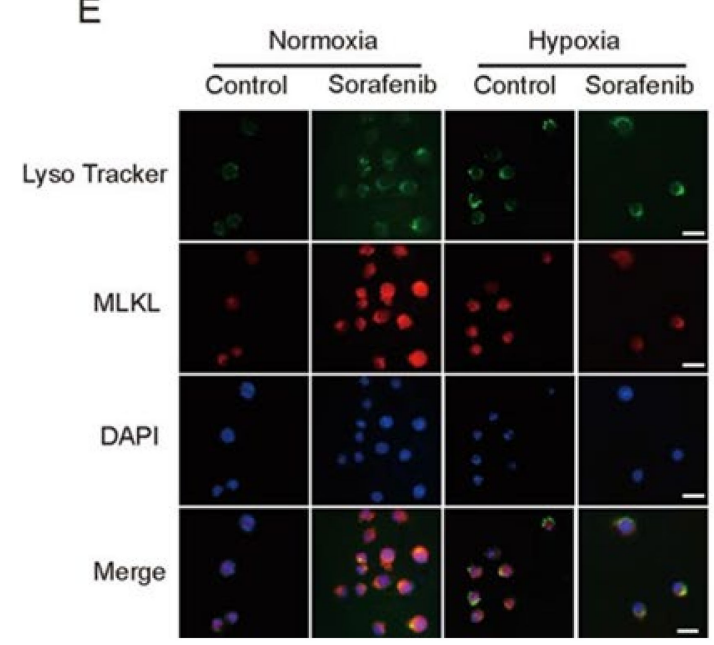

F

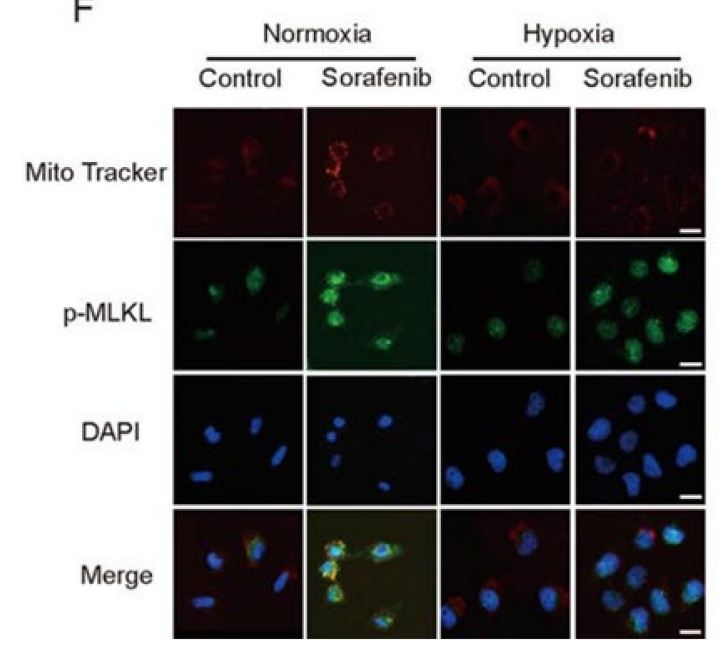

Figure 3. Hypoxia Impeded the Distribution of RIPK1/RIPK3/MLKL Complex in Cytoplasm. (A) Distribution of necroptosis proteins in cytoplasmic and nuclear observed by WB. (B) Distribution of RIPK1/RIPK3/MLKL complex in cytoplasm by Co-IP. (C-F) Expression of MLKL on different organelles, which has a rich membrane structure such as a cell membrane, mitochondria, endoplasmic reticulum, and lysosome observed by immunofluorescence. (C): Scale bar $=80 \mu \mathrm{m}$. (D-F): Scale bar $=40 \mu \mathrm{m}$. 
A

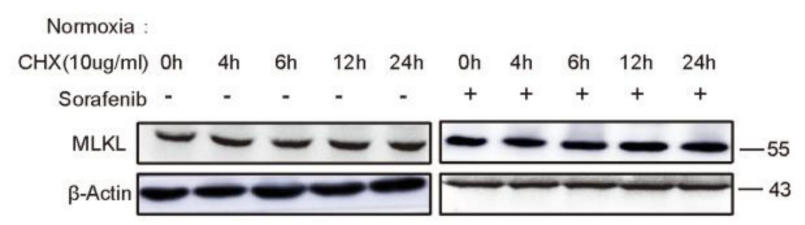
Chloroquir
Hypoxia

$\mathrm{CHX}(10 \mathrm{ug} / \mathrm{ml})$ Oh $\quad 4 \mathrm{~h} \quad 6 \mathrm{~h} \quad 12 \mathrm{~h} \quad 24 \mathrm{~h} \quad$ Oh $\quad 4 \mathrm{~h} \quad 6 \mathrm{~h} \quad 12 \mathrm{~h} \quad 24 \mathrm{~h}$ Sorafenib -

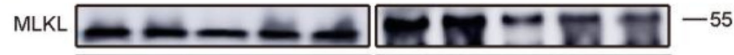

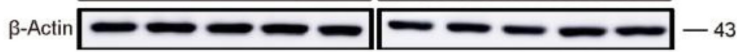

$\mathrm{CHX}(10 \mathrm{ug} / \mathrm{ml})$

ne (50uM) Oh $\quad 4 \mathrm{~h} \quad 6 \mathrm{~h} \quad 12 \mathrm{~h} \quad 24 \mathrm{~h} \quad$ Oh $\quad 4 \mathrm{~h} \quad 6 \mathrm{~h} \quad 12 \mathrm{~h} \quad 24 \mathrm{~h}$

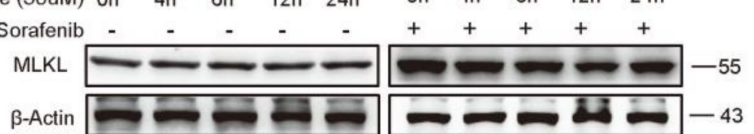

B

C

Normoxia Hypoxia
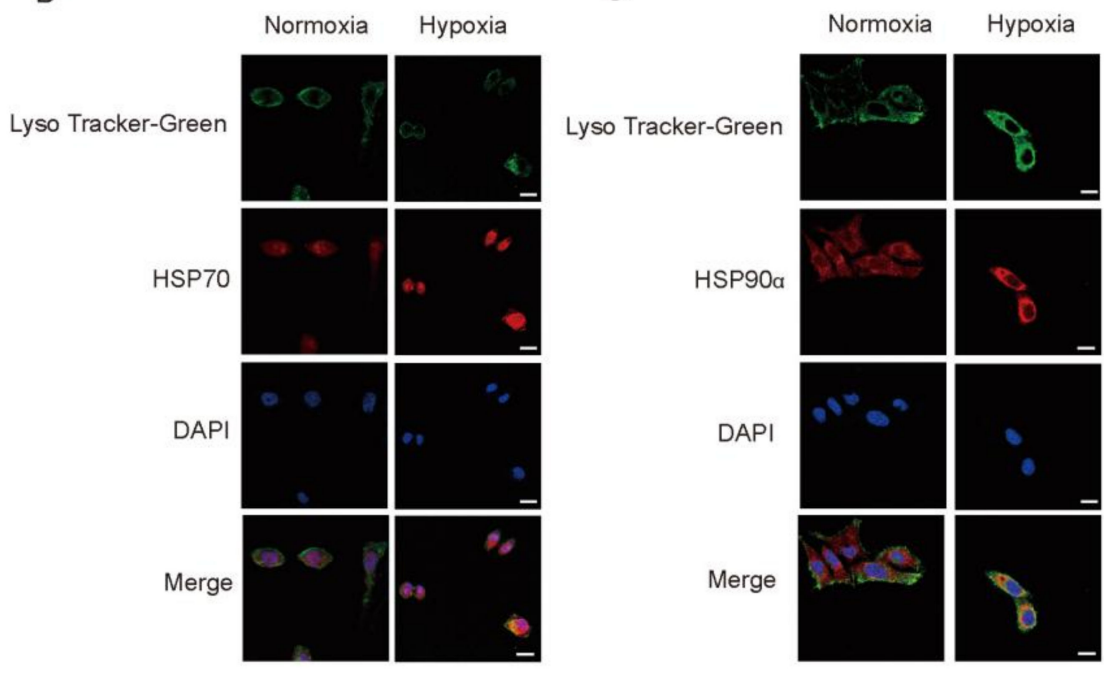

D

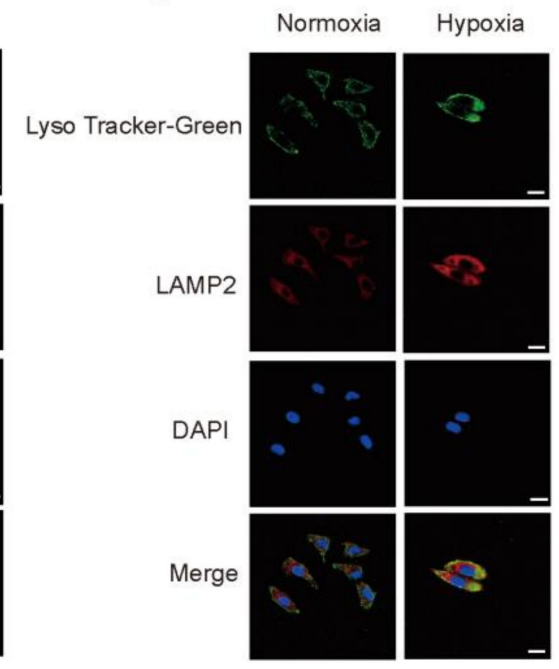

E

F
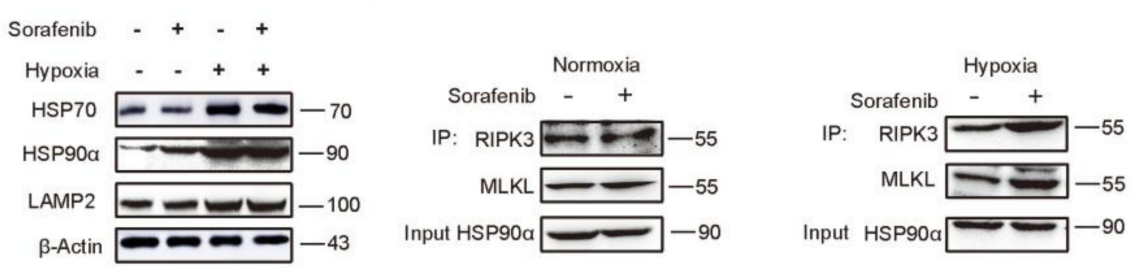

G

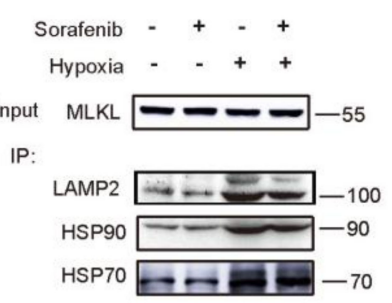

$\mathrm{H}$

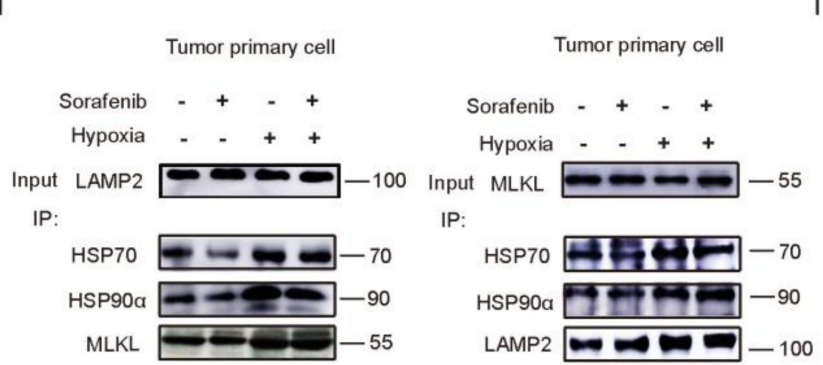

I

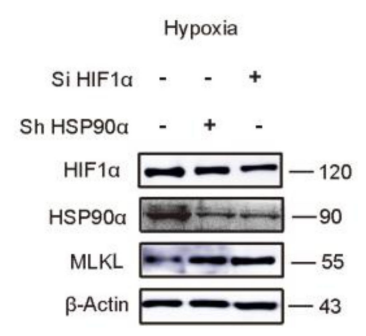

J

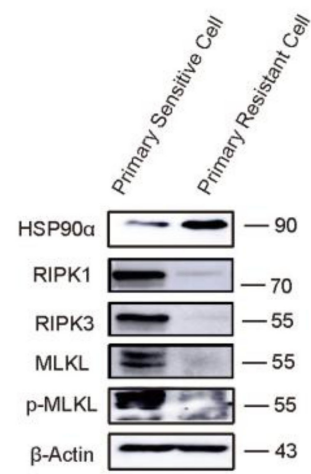

Figure 4. HSP90 $\alpha$ Promotes Chaperone-Mediated Autophagy (CMA) Degradation by directly binding to MLKL in hypoxia. (A) The degradation stability of MLKL detected by Western blot. (B-D) The expressions of LAMP2, HSP90 $\alpha$ and HSP70 located in lysosomal observed by immunofluorescence. Scale bar $=40 \mu \mathrm{m}$. (E) The expression of LAMP2, HSP90 $\alpha$ and HSP70 in hypoxia. (F) HSP90 $\alpha$ binding with necroptosis protein. (G,H) The expression of LAMP2/HSP90 $\alpha / H S P 70 / M L K L$ complex detected by Western blot in HepG2 and primary tumor cells. (I) Silenced HIF-1 $\alpha$ and HSP90 $\alpha$ affected necroptosis in hypoxia. (J) Necroptosis pathway in primary resistant cells detected by Western blot. 
Chaperone-mediated autophagy (CMA) is a lysosomal pathway of proteolysis that is responsible for the degradation of 30\% of cytosolic proteins [31,32]. HSP70 and HSP90 is the most essential component for protein transport across the lysosomal membrane in process of CMA [33,34]. They cannot only locate the substrate protein, but also recognize substrate protein. HSP90 $\alpha$ can also be formed a polymer structure on the membrane of lysosome cavity side to maintain the stability of the transport complex. LAMP2/HSP70/HSP90 $\alpha$ complex is the marker and finally identified as the lysosomal membrane receptor of CMA pathway to combine and transport substrate proteins. In Figure $4 \mathrm{~B}-\mathrm{D}$, we observed lysosomes with lyso-tracker and detected the expression of HSP70, LAMP2, and HSP90 $\alpha$ by immunofluorescence. Notably, compared normoxia, HSP70, LAMP2, and HSP90 $\alpha$, they were strikingly increased under hypoxia and were located in lysosomes. Similarly, WB results indicated that HSP70, LAMP2, and HSP90 $\alpha$ were high expressed under hypoxia (Figure 4E). Furthermore, the capacity of HSP90 $\alpha$ binding with RIPK3 and MLKL was much more in hypoxia than that in normoxia (Figure 4F). The HSP70/HSP90 $\alpha /$ LAMP2/MLKL complex was also detected by Co-IP. We tested the capacity of MLKL binding with HSP70/HSP90 $\alpha$ /LAMP2 being increased obviously under hypoxia conditions in HepG2 cells (Figure 4G). Similar results were found in primary tumor cells (Figure 4H). Besides, the capacity of LAMP2 binding with HSP70/HSP90 $\alpha /$ MLKL was increased in hypoxia. LAMP2 and HSP90 $\alpha$ were also highly expressed in the tumor internal hypoxia area compared to the normoxia tumor area in vivo; however, the expression of MLKL was decreased in hypoxia (Figure S2F). Collectively, MLKL, as a customer, was recognized by the HSP70/HSP90 $\alpha$ /LAMP2 transporter and was transported into lysosome, and then degraded in the end; all these results suggested that MLKL could be degraded under hypoxia through the chaperone-mediated autophagy (CMA) degradation pathway.

To test the role of HSP90 $\alpha$ in the necroptosis pathway, siRNA was used. As shown in Figure 4I, there was a loss of HIF1 $\alpha$ inhibited HSP90 $\alpha$, while a loss of HSP90 $\alpha$ did not change the HIF1 $\alpha$ expression. Besides, both inhibition of HIF $1 \alpha$ and HSP $90 \alpha$ can promote MLKL recovered under hypoxia. In Figure $4 \mathrm{~J}$, necroptosis related proteins were downregulated obviously and HSP90 $\alpha$ was highly expressed in primary resistant cells, which suggested that necroptosis was dull in Sorafenib-resistant tumor cells. Taken together, these results indicated that HSP90 $\alpha$ played a direct and important role in Sorafenib resistance by blocking necroptosis.

\subsection{7-AAG Combining with Sorafenib Enhanced Necroptosis Pathway In Vitro}

In order to study whether the inhibitory effect of HSP90 $\alpha$ can improve the necroptosis induced by Sorafenib, 17-AAG, an inhibitor of $\mathrm{HSP} 90 \alpha$, was used. As shown in Figure 5A, when combined with $1 \mu \mathrm{M}$ 17-AAG, the PI-positive cells were increased to $29.6 \%$ and $37.7 \%$, respectively in HepG2 and Huh7. While in Sorafenib group, only about $10.73 \%$ and $19.6 \%$ of dead cells were increased in hypoxia. 17-AAG showed low effects on HCC cells when used alone.

MTT results in Figure 5B also showed that hepatoma cell lines were insensitive to Sorafenib in hypoxia; the survival of HepG2 and Huh7 was $41.08 \%$ and $36.4 \%$, respectively, when 17-AAG was combined with Sorafenib. In general, the inhibitory effect of Sorafenib was increased significantly under hypoxia when 17-AAG was used. In Figure 5C, 17-AAG inhibited HSP90 $\alpha$ and safeguard necroptosis induced by Sorafenib. Immunofluorescence presented a similar phenomenon in HCC cell lines (Figure 5D). All the results revealed that 17-AAG reversed the Sorafenib resistance in hypoxia and enhanced the inhibitory effect of Sorafenib on HCC. 
A

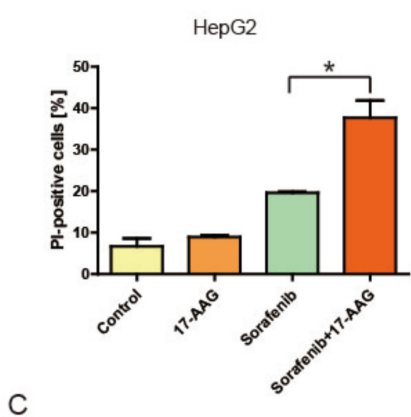

C

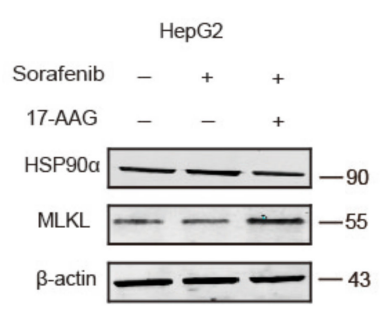

Huh7

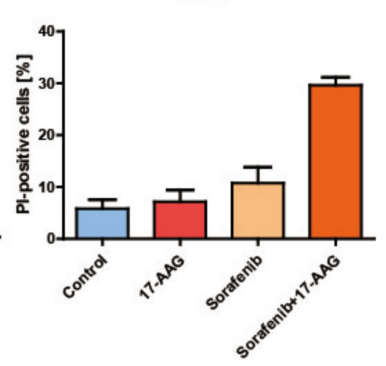

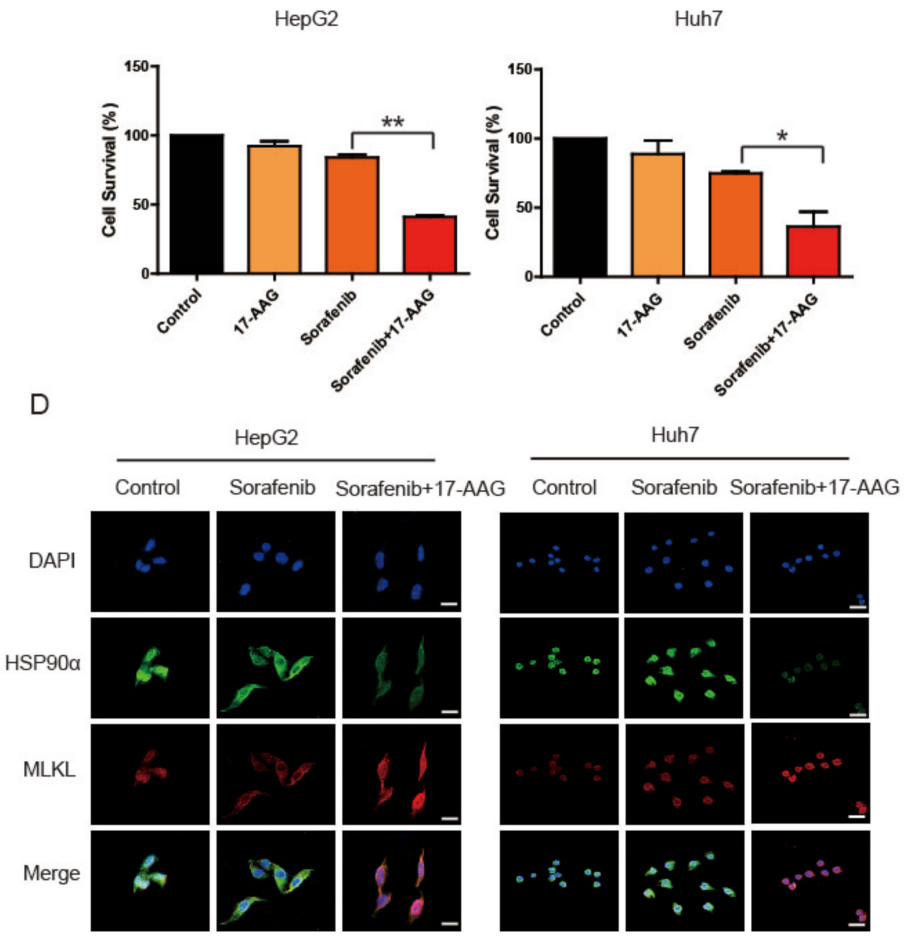

Figure 5. 17-AAG Combined with Sorafenib Treatment Enhanced Necroptosis Pathway in vitro. (A) Detection of death cells under hypoxia by Flow Cytometry. The results were shown as mean \pm SEM from three independent experiments. ${ }^{*} p<0.05,{ }^{* *} p<0.01$. (B) The survival of liver cancer cells under hypoxia assessed by the MTT assay. The results were shown as mean \pm SEM from three independent experiments. ${ }^{*} p<0.05,{ }^{* *} p<0.01$. (C,D) The detection of HSP90 $\alpha$ and MLKL by Western blot and Immunofluorescence. Scale bar $=40 \mu \mathrm{m}$.

\subsection{HSP90 $\alpha$ Could Be an Important Target in Sorafenib Resistance In Vivo}

To further verify the pivotal role of HSP90 $\alpha$ in Sorafenib Resistance in vivo, the HSP90 $\alpha$ knockdown primary cell line was established. In the Sorafenib resistant xenograft model, we tested the effect of 17-AAG combined with Sorafenib and HSP90 $\alpha$ knockdown combined with Sorafenib, the results indicated that Sorafenib reduced the tumor growth most significantly when HSP90 $\alpha$ was inhibited (Figure 6A-C).

Compared to the control group, the tumor weight has also been significantly reduced, to $57 \%$ and $48 \%$ in the $17-A A G$ combined Sorafenib group and Sorafenib combined HSP $90 \alpha$ knockdown group, respectively. While the Sorafenib alone has poor efficacy in resistant tumor. The expression of HIF1 $\alpha /$ HSP90 $\alpha$ and RIPK3/MLKL were also detected by immunofluorescence and IHC tissue sections (Figure 6D,E). Necroptosis related proteins were low expressed in resistant tumors and HIF1 $\alpha / \mathrm{HSP} 90 \alpha$ was highly expressed. However, in ShHSP90 $\alpha$ group, necroptosis induced by Sorafenib was surprisingly reactivated. All these results indicated that HSP90 $\alpha$ can be a useful target of Sorafenib resistance therapy via recovering the necroptosis pathway in liver cancer.

\subsection{Clinical Analysis of HIF $1 \alpha / H S P 90 \alpha$ as a Therapeutic Target}

Results of Figure 7A showed that the overall survival of HSP90 $\alpha$, HIF-1 $\alpha$, RIPK1 and RIPK3 in Kaplan-Meier Plotter. 
A

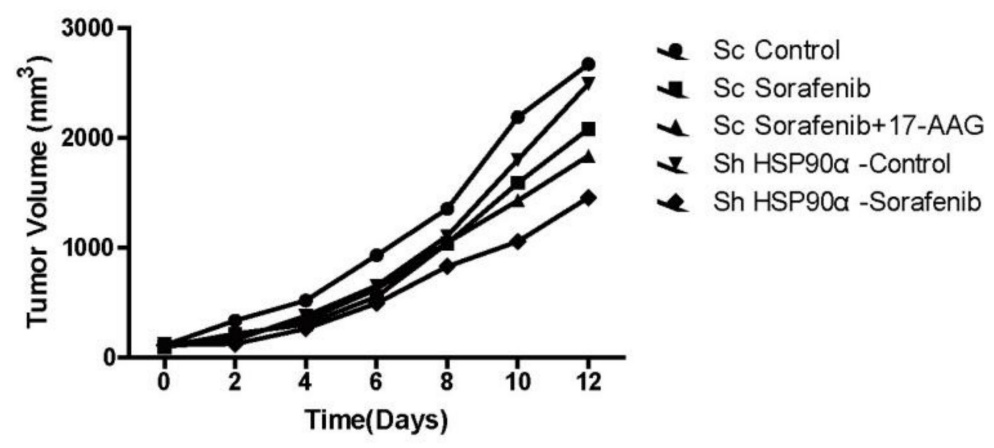

B

C

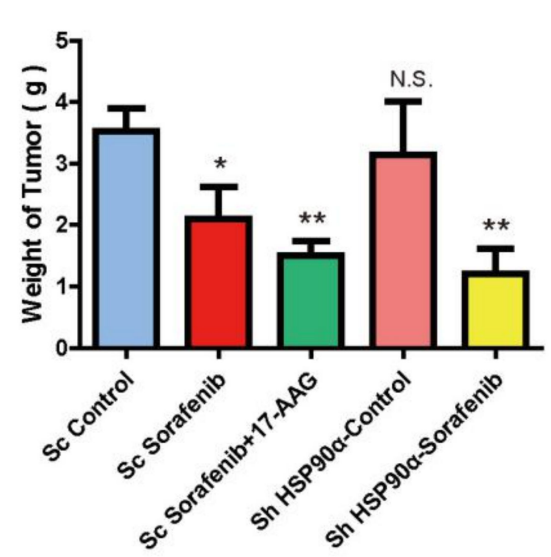

D

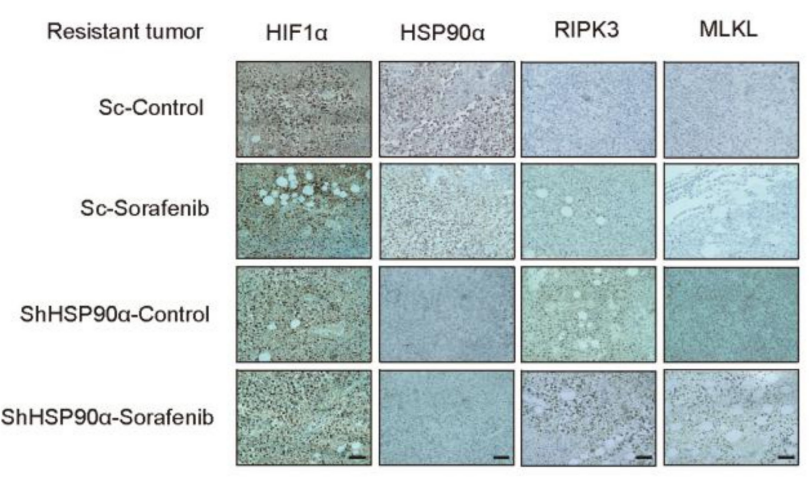

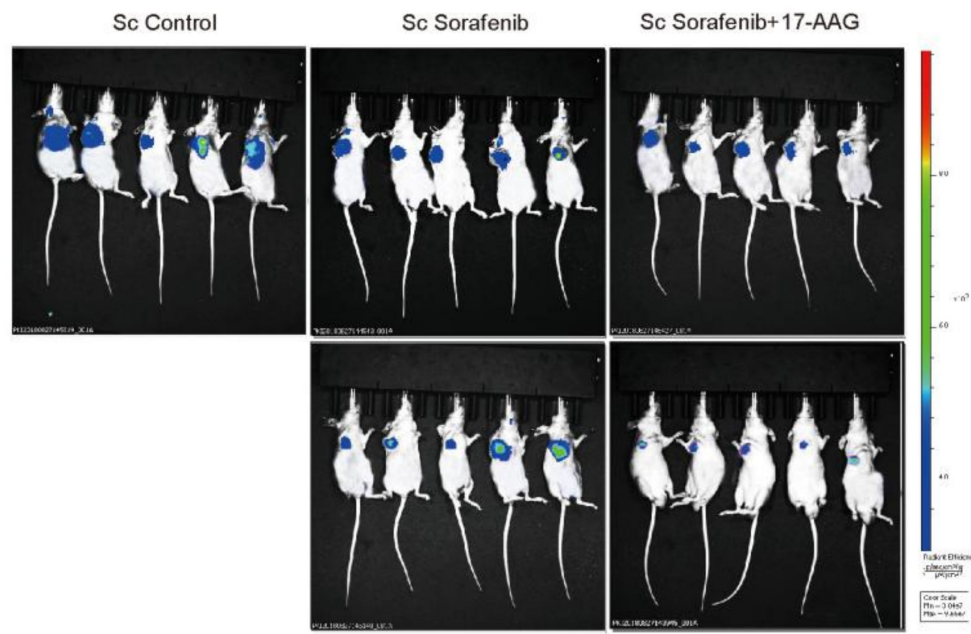

Sh HSP90a-Control

Sh HSP90a-Sorafenib

E

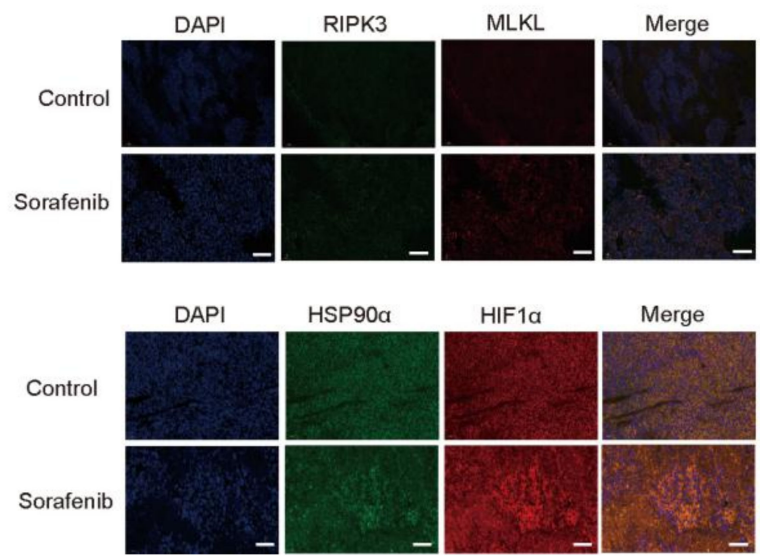

Figure 6. HSP90 $\alpha$ can be a Useful Target in Sorafenib Resistance Therapy In Vivo. (A) Tumor volume was recorded in primary Sorafenib resistant xenograft model. (B) Tumor weight was recorded in primary Sorafenib resistant xenograft model. The results were shown as mean \pm SEM from three independent experiments. ${ }^{*} p<0.05,{ }^{* *} p<0.01$, ns, not significant. (C) Bioluminescence images of the primary Sorafenib resistant xenograft model. (D,E) The expression of HIF1 $\alpha$, HSP90 $\alpha$ and necroptosis pathway in tumors detected by IF and IHC. Scale bar $=800 \mu \mathrm{m}$. 
A

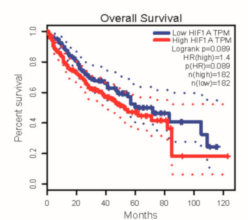

HIF1a

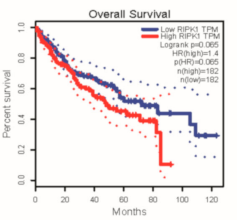

RIPK1

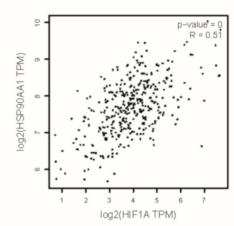

$E$
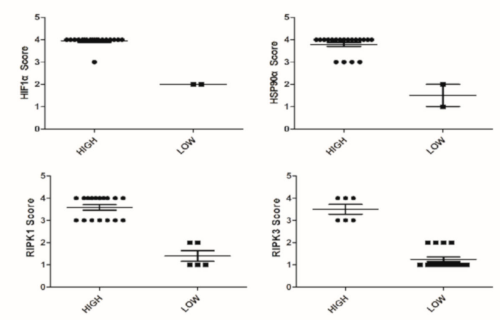

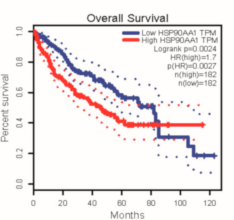

HSP90a

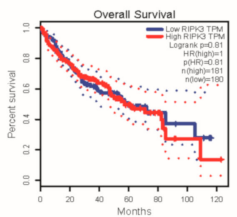

RIPK3
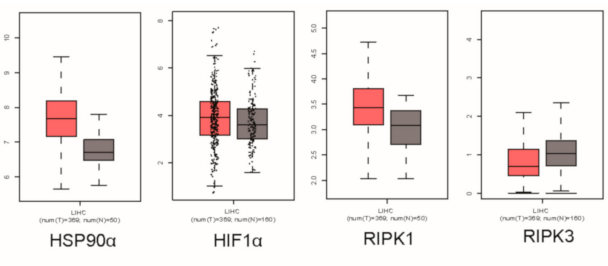

D

Normal tissue

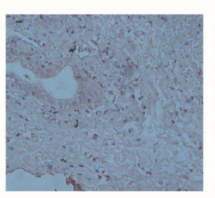

Paraplastic tissue

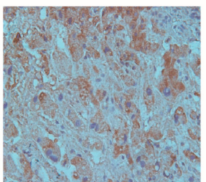

Tumor tissue

HSP90a
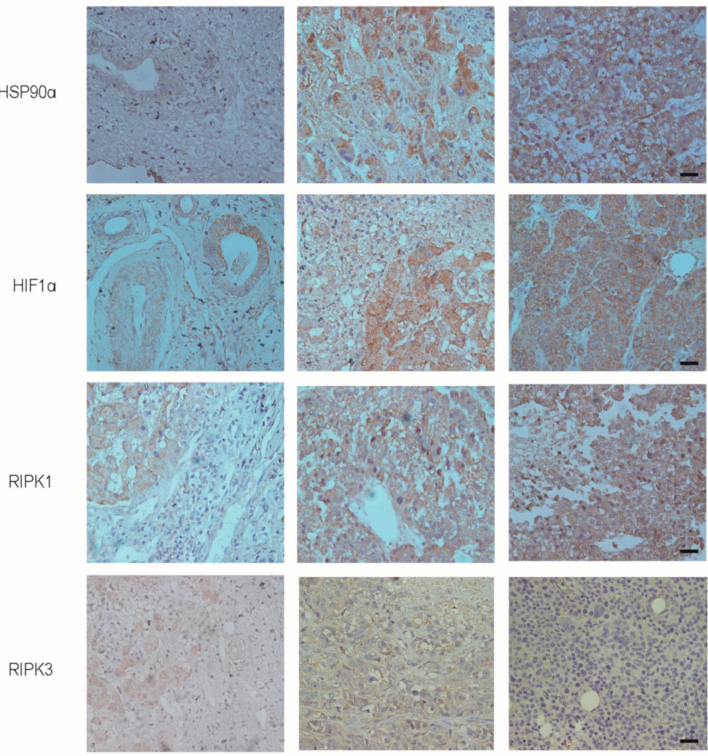

RIPK

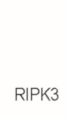

LAMP2
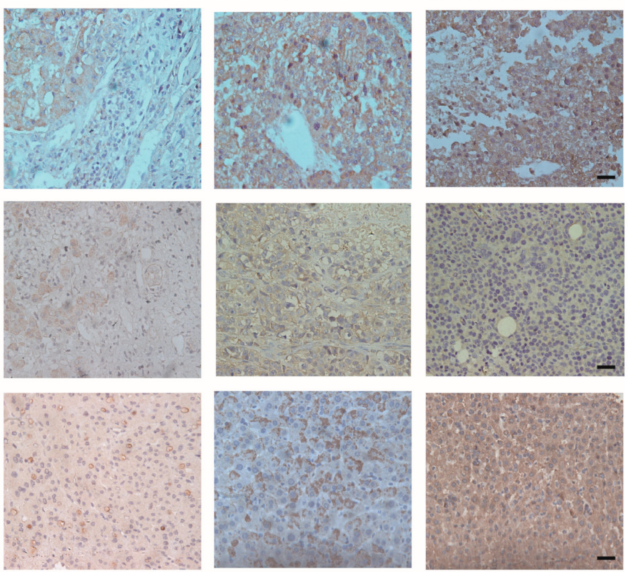

F

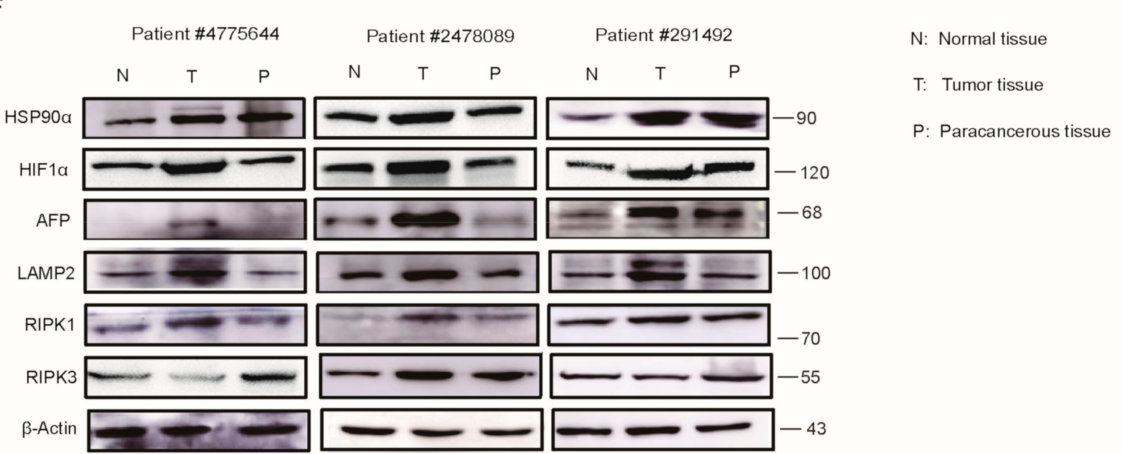

Figure 7. Clinical Analysis of HIF1 $\alpha / \mathrm{HSP} 90 \alpha$ as a Therapeutic Target. (A,B) The overall survival and correlation of several proteins in GEPIA (Gene Expression Profiling Interactive Analysis) database. (C) Expressions of HSP90 $\alpha$, HIF-1 $\alpha$, RIPK1 and RIPK3 in GEPIA (Gene Expression Profiling Interactive Analysis) database. (D) Protein expressions in tumor, para-carcinoma, and normal tissues of patient samples detected by IHC. Scale bar $=500 \mu \mathrm{m}$. (E) Analysis the expressions of HSP90 $\alpha$, HIF-1 $\alpha$, RIPK1 and RIPK3 in patient samples. (F) Protein expressions in tumor, para-carcinoma, and the normal tissues of patient samples detected by WB.

The database showed that the higher the HIF1 $\alpha / \mathrm{HSP} 90 \alpha$ expression, the worse the patient prognosis. It looks like RIPK1 has a short survival when highly expressed and the expression of RIPK 3 has no obvious effect on the survival of liver cancer. The HSP $90 \alpha$ are the most significant of these genes, the $\mathrm{p}$ value is 0.0027 . Additionally, HIF $1 \alpha / \mathrm{HSP} 90 \alpha$ showed positive correlation in the GEPIA (Gene Expression Profiling Interactive Analysis) database (Figure 7B). The expression of HSP90 $\alpha$, HIF1 $\alpha$ and RIPK1 was stronger in cancer tissues than in liver tissues; however, RIPK3 was expressed more in liver tissues (Figure 7C). RIPK1 may get close to cancer development. The role of RIPK3 in cancer development is 
still unclear. We also analyzed the samples of patients with liver cancer by IHC and WB in Figure 7D-F, and similar results were obtained. HIF1 $\alpha /$ HSP90 $\alpha$ and RIPK1 were almost highly expressed in liver cancer tissues. We found that, in most cases, the expression of RIPK3 in cancer tissues was lower than that in normal tissues, and there are still about $25 \%$ cases showing the opposite situation. In general, we believe that RIPK3 may have a negative regulatory effect on tumorigenesis, different from RIPK1, and it has been reported in the reference that RIPK1 is involved in the NF- $\mathrm{KB}$ signaling pathway to regulate cancer development. We think that the specific effects of the necroptotic signaling pathway on liver cancer requires more research. As a molecular chaperone, HSP $90 \alpha$ plays important roles in proliferation, apoptosis, and the drug resistance of various biological behaviors. It is a regulator of P53, HIF1 $\alpha, \mathrm{P} 23, \mathrm{HSF}$ and so on, which can perform their functions in the development of cancer. HSP90 $\alpha$ can not only regulate the HIF signaling pathway but also plays a crucial role in the necroptosis signaling pathway. As a systemic expression gene, HSP $90 \alpha$ plays an important role in proliferation, apoptosis, and drug resistance of various biological behaviors, and it is a regulator of other proteins that can perform their functions. All these results suggested that $\mathrm{HSP} 90 \alpha$ would be a promising and useful target in hepatocellular carcinoma therapy.

\section{Discussion}

At present, the effective treatment of liver cancer is still very limited and the recurrence rate of liver cancer is still high. Sorafenib remains the only FDA-approved systemic drug for patients with advanced HCC. In fact, both the direct inhibitory effect of Sorafenib on tumor cells and on angiogenesis are very important. Many articles summarized mechanisms of Sorafenib. Sorafenib suppresses tumor angiogenesis and proliferation, and induces tumor cell apoptosis. The emergence of Sorafenib resistance is a gradual process. After a long-term use of Sorafenib, ischemic and hypoxic will appeared in tumor area due to the inhibition of microvessels. The inhibition of Sorafenib on tumor cells is weakened, and then drug resistance is further developed. In our study, we found that MLKL was degraded under hypoxia. Necroptosis was blocked, which results in Sorafenib resistance. The understanding of the mechanism of Sorafenib resistance will enable us to use it more effectively in clinics.

Here, we demonstrated that necroptosis would be one of the main targets of Sorafenib on HCC. Necroptosis related proteins RIPK1, RIPK3 and MLKL were highly expressed in hepatocellular carcinoma cells and tumor tissues with Sorafenib treatment, which means that Sorafenib activated the necroptosis pathway in vitro and in vivo. Hypoxia is a hallmark of solid tumors due to the rapid growth of cancer cells and the abnormal angiogenesis. Recent studies have confirmed that the adaptation of tumors to hypoxic microenvironment is not only to maintain the survival or growth of tumors, but also play an important role in drug resistance. In the present study, we demonstrated that hypoxia rendered resistance to Sorafenib in human HCC cells by attenuating necroptosis. We observed that abundant HSP90 $\alpha$ binds with necrosome directly, and MLKL was found degraded by autophagy lysosomal degradation pathway in hypoxia. Further study revealed that the expressions of HSP70/HSP90 $\alpha /$ LAMP2/MLKL complex was increased under hypoxia and was located in lysosomes. Therefore, all our results presented that MLKL was degraded under hypoxia, which resulted in the decreasing of the RIPK1/RIPK3/MLKL complex. Then necroptosis was interrupted and drug resistance appeared. Clinical data and patient samples also suggest that HSP90 $\alpha$ expression in hepatocellular carcinoma was associated with prognosis and Sorafenib resistance. The blockage of $\mathrm{HSP} 90 \alpha$ can significantly overcome Sorafenib resistance under hypoxia in vitro and in vivo.

17-AAG, a derivative of geldanamycin, is currently undergoing clinical development as a novel anticancer agent for the treatment of human cancers. It has been reported that 17-AAG could be an effective anticancer drug, whether used alone or in combination with other drugs [35,36]. Here, we found that 17-AAG decreased $\mathrm{HSP} 90 \alpha$, and then safeguarded the activated necroptosis fluently. 17-AAG combining with Sorafenib showed 
great inhibitory effects in HCC in vivo and in vitro. In fact, 17-AAG combined with Sorafenib increased both apoptosis and necroptosis. Besides, many researchers have proved that 17-AAG could inhibit cell proliferation, tumor metastasis and inducing cell apoptosis in many cancers; in this study, we focus on 17-AAG combined with Sorafenib, thus enhancing the necroptosis of HCC. Overall, our study demonstrates that Sorafenib induced necroptosis is a considerable pathway in liver cancer and HSP $90 \alpha$ plays a critical role in Sorafenib resistance under hypoxia by blocking necroptosis. 17-AAG combined with Sorafenib is a promising therapy for hepatocellular carcinoma.

\section{Materials and Methods}

\subsection{Reagents}

Sorafenib (Bay 43-9006, Sigma-Aldrich), 17-AAG (Tanespimycin, MCE, HSP90 $\alpha$ inhibitor), Z-VAD-FMK (Selleck, Caspase Inhibitor), Necrostatin-1 (Selleck, RIPK1 inhibitor), Necrosulfonamide (Selleck, MLKL inhibitor).

MTT (3-(4,5-dimethylthiazol-2-yl)-2,5-diphenytetrazoliumbromide) was obtained from Fluka Chemical Corp (Ronkonkoma, NY, USA) and was dissolved in $0.01 \mathrm{M}$ phosphatebuffered saline (PBS). Antibodies against RIPK1(A7414), RIPK3(A5431), MLKL(A5579), HIF-1 $\alpha$ (A16873) were purchased from Abclonal Technology (Wuhan, China). Antibodies against, HSP90 $\alpha$ (BS6461), were products of Bioworld Technology (USA). Antibodies against AFP (4448) and LAMP2 (49067) were products of Cell Signaling Technology (Beverly, MA, USA). Antibodies against Lamin A (sc-293162), $\beta$-Tubulin (sc-166729) and $\beta$-Actin (sc-8432) were products of Santa Cruz Biotechnology (USA). Normal mouse and rabbit IgG-HRB secondary antibodies were purchased from Santa Cruz Biotechnology.

\subsection{Cell Culture}

In this study, different human HCC cell lines (Huh7, HepG2) were purchased from the Shanghai Institute of Cell Biology, Chinese Academy of Sciences (Shanghai, China). The human HCC cells were cultured in Dulbecco's Modified Eagle Medium (Gibco, Carlsbad, CA, USA) with $10 \%(v / v)$ heat-inactivated fetal bovine serum (Gibco, Paisley, Scotland), $100 \mathrm{U} / \mathrm{mL}$ streptomycin and $100 \mathrm{U} / \mathrm{mL}$ penicillin at $37^{\circ} \mathrm{C}$ with $5 \% \mathrm{CO}_{2}$. Primary tumor cells were isolated from tumor tissues and were cultured with the special medium for primary human liver cancer culture medium (iCell Bioscience Inc, Shanghai) with 10\% fetal bovine serum.

Cells were maintained at $37^{\circ} \mathrm{C}$ in a humidified incubator containing $20 \% \mathrm{O}_{2}, 5 \% \mathrm{CO}_{2}$ and $75 \% \mathrm{~N}_{2}$ in normoxia. The hypoxic condition was achieved at $37{ }^{\circ} \mathrm{C}$ with a gas mixture containing $1 \% \mathrm{O}_{2}, 94 \% \mathrm{~N}_{2}$ and $5 \% \mathrm{CO}_{2}$ in a humidified atmosphere.

\subsection{Clinical Samples}

Fresh primary liver cancer tissue and adjacent nontumor liver tissue samples were obtained from HCC patients undergoing hepatectomy, hepatic tumor ablation or percutaneous transhepatic biopsy and similar liver surgery at Jiangsu Cancer Hospital and NanJing Drum Tower Hospital.

\subsection{PDX (Patient-Derived Tumor Xenograft) Model and Sorafenib—Resistance Model In Vivo}

PDX model was constructed by the transplantation of tumor tissue from patients into severe NSG immunodeficiency mice; 5-6-week-old NSG mice were purchased from Vital River Laboratory Animal Technology (Beijing, China). Liver cancer samples were inserted into the mice's armpit, and were then fed with normal feed and drinking water. As shown in Figure S3A, when the tumor size reached $400-500 \mathrm{~mm}^{3}$, tumor tissues were stripped and inoculated into other immunodeficiency mice, which was the first generation. For the inducing resistant group, when the tumor size reached $100 \mathrm{~mm}^{3}$, the mice were given an oral dosage of Sorafenib $80 \mathrm{mg} / \mathrm{kg}$ once a day. At the fourth generation, the Sorafenib resistance was detected obviously. Data in Figure S3B showed the successive processes of Sorafenib inducing resistance. The sensitive primary cells were extracted 
from the tumors of non-treated group. The resistant primary cells were extracted from the tumors of the Sorafenib induced resistant group. The drug resistance was tested by MTT assay (Figure S3C), flowcytometry (Figure S3D), and trypan blue staining (Figure S3E). The morphology of primary resistant cells was also observed by Transmission Electron Microscope. Sorafenib could not induce cell death, including necroptosis in primary resistant cells (Figure S3F).

\subsection{Animal Studies}

5-6-week-old female BALB/c nude mice were purchased from the SLAC Laboratory (Shanghai, China). The transplanted tumors were induced by subcutaneous injection into the flanks of the mice with $3.0 \times 10^{6} / 0.1 \mathrm{~mL}$ HepG2, Huh7 cells or primary tumor cells. A couple of days later, the tumor volume was measured by micrometer calipers, according to the size of the tumor, and the mice were average divided into groups. Additionally, mice were treated with $60 \mathrm{mg} / \mathrm{kg}$ Sorafenib every 2 days orally. Nec $(1.65 \mathrm{mg} / \mathrm{kg})$ and 17-AAG $(25 \mathrm{mg} / \mathrm{kg}$ ) was administered intra-peritonelly twice a week. 3 weeks later, the mice were killed, and the tumor xenografts were removed and measured. Tumor volume (TV) was calculated using the following formula: TV $\left(\mathrm{mm}^{3}\right)=\mathrm{D} / 2 \times \mathrm{d}^{2}$, where $\mathrm{D}$ is the longest diameter and $\mathrm{d}$ is the shortest diameters.

All experiment animals were raised in air-conditioned rooms under controlled lighting (12 h light/day) and were provided with food and water at our discretion. Animal care and surgery protocols were approved by the Animal Care Committee of China Pharmaceutical University. All the animals were treated and used in a scientifically valid and ethical manner.

\subsection{Western Blot Analysis}

Cells were washed with cold PBS and lysed in RIPA Lysis buffer (ThermoFisher, ShangHai, China) containing protease/phosphatase inhibitors. After the lysates concentration was determined by the BCA protein assay (Pierce, Rockford, IL, USA), an equal amount of denatured proteins were subjected to SDS-PAGE gel electrophoresis and then transferred onto a nitrocellulose membrane (PallCor, Arroyto, Cordoba, Argentina), which was blocked by $5 \%$ non-fat milk in PBS, followed by incubation at $4{ }^{\circ} \mathrm{C}$ with specific primary antibodies overnight. Then, membranes were incubated with HRP goat anti-rabbit immunoglobulin $\mathrm{G}(\mathrm{IgG} ; \mathrm{H}+\mathrm{L})$ or anti-mouse IgG $(\mathrm{H}+\mathrm{L})$ secondary antibody (Biosharp) for $1 \mathrm{~h}$ and finally visualized with chemiluminescence (ThermoFisher). Immunoreactive proteins were detected with the Tanon-5200 Multi Automatic chemiluminescence/fluorescence image analysis system (Tanon-5200, Tanon). All uncropped images were shown in Figures S4-S6.

\subsection{Immunohistochemistry (IHC)}

Paraffin embedding sections were heated at $60^{\circ} \mathrm{C}$ for half an hour and were dewaxed. For the IHC assay, tissue sections were incubated with $0.3 \%$ Triton-X 100 for 20 min after antigen retrieval. Then, the solution containing goat serum was used to block nonspecific binding sites. Next, primary antibodies were added and incubated at $4{ }^{\circ} \mathrm{C}$ overnight. Before interacting with DAB solution, tissues were incubated with biotin-labeled secondary antibodies at room temperature for $30 \mathrm{~min}$. Finally, tissues were stained with hematoxylin and covered by neutral gum - the process was performed with standard techniques. The immunohistochemistry kit was purchased from Shanghai Yeasen BioTechnologies co., Ltd.

\subsection{Immunofluorescence Assay}

The cells were seeded onto cover glasses in a 6-well plate and fixed by $4 \%$ PFA for $15 \mathrm{~min}$, followed by permeabilization using $0.3 \%$ Triton-X 100 for $15 \mathrm{~min}$. Then, the cover glasses were blocked with $3 \%$ bovine serum albumin for $1 \mathrm{~h}$ and incubated with primary antibody at $4{ }^{\circ} \mathrm{C}$ overnight. Then, the cells were incubated with Alexa Fluor-conjugated secondary antibodies (Invitrogen, Carlsbad, CA, USA) for $1 \mathrm{~h}$ and stained by DAPI for $20 \mathrm{~min}$. The confocal microscope was employed to photograph the protein expressions 
or location in the cells. Various organelle fluorescent probes such as ER-Tracker (KeyGen Biotech, NanJing, China) and Mito-Red (KeyGen Biotech, NanJing, China) were incubated before paraformaldehyde was fixed and the next steps were the same as above. After 30 min incubation at $37^{\circ} \mathrm{C}$ in the dark, coverslips were fixed in $4 \%$ paraformaldehyde for $30 \mathrm{~min}$. The images were captured with the Olympus FV1000 confocal microscope.

\subsection{Cell Transfection}

HIF1- $\alpha$ siRNA was purchased from Santa Cruz Biotechnology. SiRNA transfections were performed according to the manufacturer's instructions, using Lipofectamine 2000 reagent (Invitrogen). After that, the transfected system was removed, and it was not until $24 \mathrm{~h}$ cultured in normal media that the cells were used for further experiment.

To establish HSP90 $\alpha$-knockdown cells, 293T cells were transfected with lentivirus plasmid, together with psPAX2 and pMD2G packing plasmids by X-treme GENE 9 (Roche) for $12 \mathrm{~h}$ Fresh, with medium added to 293T cells to replace the previous medium. Then, the supernatants of $293 \mathrm{~T}$ cells were collected and mixed with fresh medium to infect tumor primary cells, along with $8 \mu \mathrm{g} / \mathrm{mL}$ polybrene. On the next day, supernatants were replaced with fresh medium containing $2 \mu \mathrm{g} / \mathrm{mL}$ puromycin. $2 \mu \mathrm{g} / \mathrm{mL}$ of concentration was maintained for more than one week and the puromycin-resistant cells were isolated and used for further experiments.

\subsection{Cytoplasmic and Nuclear Protein Extraction}

The cells were normally collected and the cells were added with buffer A and mixed evenly. After that, the cells were cracked on ice for $15 \mathrm{~min}$, and were mixed with intermittent shocks. At $4{ }^{\circ} \mathrm{C}, 13,000 \mathrm{rmp}, 5 \mathrm{~min}$, the transferred supernatant was stored as the cytoplasmic part, washed three times with buffer $\mathrm{A}$, and after washing the residual, the transferred supernatant was lysed with buffer B for $10 \mathrm{~min}$. At $4{ }^{\circ} \mathrm{C}, 13,000 \mathrm{rmp}, 10 \mathrm{~min}$, the transferred supernatant was the nuclear component; the protein concentration was measured by $\mathrm{BCA}$, and after protein denaturation, it was stored with $-20^{\circ} \mathrm{C}$ or subsequent WB experiments.

\subsection{Co-Immunoprecipitation (Co-IP)}

The nuclear extracts were incubated with $1 \mu \mathrm{g}$ control anti-IgG and $20 \mu \mathrm{L}$ of Protein A/G PLUS-Agarose (Santa Cruz) at $4{ }^{\circ} \mathrm{C}$ for $30 \mathrm{~min}$. After eliminating beads by centrifugation at $2500 \mathrm{rpm}, 10 \mu \mathrm{L}$ primary antibody was incubated with nuclear extracts at $4{ }^{\circ} \mathrm{C}$ for $1 \mathrm{~h}$ and $20 \mu \mathrm{L}$ beads were added to rotate at $4{ }^{\circ} \mathrm{C}$ overnight. On the next day, samples were centrifugated at $2500 \mathrm{rpm}$ for $5 \mathrm{~min}$ at $4{ }^{\circ} \mathrm{C}$, and then the supernatants were carefully discarded. PBS was used to wash the pellets three times, and the samples were mixed with $20 \mu \mathrm{L} 2 \times$ loading buffer and boiled for $8 \mathrm{~min}$. Finally, samples were analyzed by SDS-PAGE.

\subsection{Flowcytometry Analysis}

Cells were harvested and stained with the Annexin V/PI Cell Apoptosis Detection Kit (Vazyme Biotech Co.,Ltd, Nanjing, China) according to the manufacturer's instructions. Data were analyzed by FlowJo version 10. All the bar charts were analyzed by GraphPad Prism 6 software (GraphPad Software, San Diego, CA, USA).

\subsection{Statistical Analyses}

The data shown in the study were expressed as means \pm standard errors (SEM) from at least three independent experiments, each in triplicate samples for individual treatments or dosages. Statistical analyses were performed using ANOVA, coupled with a post hoc test.

\section{Conclusions}

Overall, our findings identify that Sorafenib induced necroptosis in liver cancer and HSP $90 \alpha$ plays a critical role in Sorafenib resistance under hypoxia by blocking necroptosis. 
Additionally, 17-AAG, combined with Sorafenib, is a promising therapy for hepatocellular carcinoma.

Supplementary Materials: The following are available online at https:/ / www.mdpi.com/2072-669 4/13/2/243/s1, Figure S1: Necroptosis Pathway Supplementary Detection,Figure S2: MLKL related Supplementary Experiment, Figure S3: Sorafenib-Resistance Model, Figures S4-S6: uncropped WB images.

Author Contributions: Y.L., Y.Y. (Yue Yang) and L.Z. conceived the study. Y.L. and Y.Y. (Yue Yang) performed the experiments and collected the data. Y.L. analyzed the data and drafted the manuscript. Y.L., Y.Y. (Yue Yang) and L.Z. participated in the design of the study and performed the statistical analysis. Y.L., Y.Y. (Yue Yang), D.P., Y.D., H.Z., Y.Y. (Yuting Ye), J.L. and L.Z. discussed the results. Y.L., Y.Y. (Yue Yang) and L.Z. participated in its design and coordination and helped to draft the manuscript. All authors have read and agreed to the published version of the manuscript.

Funding: This study was supported by the National Natural Science Foundation of China (No. 81773774), “Double First-Class University project” of China Pharmaceutical University (CPU2018GY37).

Informed Consent Statement: Informed consent was obtained from all subjects involved in the study.

Data Availability Statement: All data generated or analysed during this study are included in this article. The data presented in this study are available on request from the corresponding author.

Acknowledgments: We appreciated all authors for their contributions to the manuscript. We also sincerely appreciated the help from Pathologh and PDX efficacy evaluation Center.

Conflicts of Interest: All the authors declare that they have no competing financial interests or personal relationships that could have appeared to influence the work reported in this paper.

Ethics Approval and Consent to Participate: The study was conducted according to the guidelines of the Declaration of Helsinki. Animal care and surgery protocols (CPU-20180309c5100342(108)) are approved by the Animal Care Committee of China Pharmaceutical University.

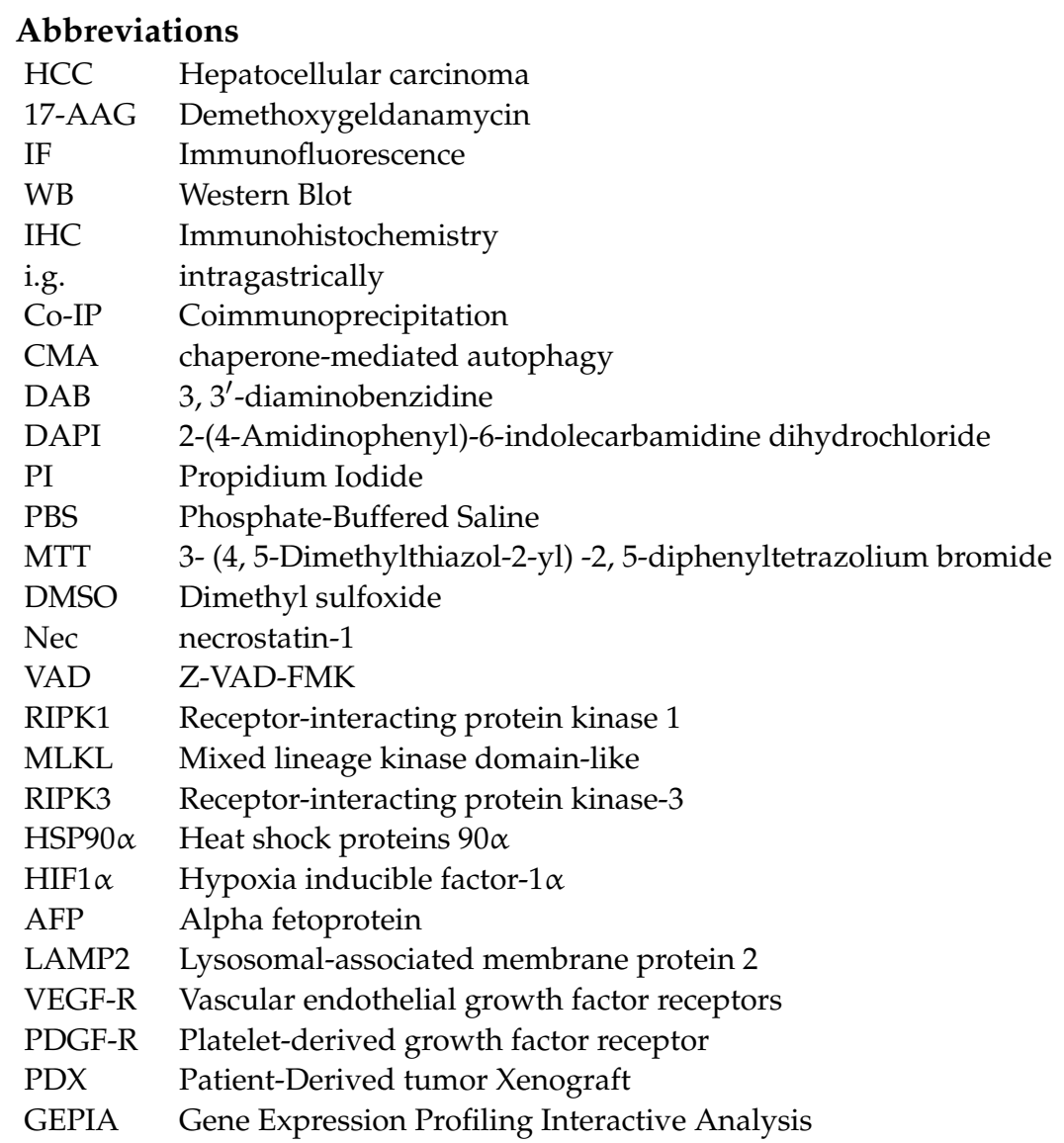




\section{References}

1. Torre, L.A.; Bray, F.; Siegel, R.L.; Ferlay, J.; Lortet-Tieulent, J.; Jemal, A. Global cancer statistics, 2012: Global Cancer Statistics, 2012. CA Cancer J. Clin. 2015, 65, 87-108. [CrossRef] [PubMed]

2. Chun, L.J.; Tong, M.J.; Busuttil, R.W.; Hiatt, J.R. Acetaminophen Hepatotoxicity and Acute Liver Failure. J. Clin. Gastroenterol. 2009, 43, 342-349. [CrossRef] [PubMed]

3. Macek-Jilkova, Z.; Kuyucu, A.Z.; Kurma, K.; Pour, S.T.A.; Roth, G.S.; Abbadessa, G.; Yu, Y.; Schwartz, B.; Sturm, N.; Marche, P.N.; et al. Combination of AKT inhibitor ARQ 092 and sorafenib potentiates inhibition of tumor progression in cirrhotic rat model of hepatocellular carcinoma. Oncotarget 2018, 9, 11145-11158. [CrossRef] [PubMed]

4. Zhai, B.; Hu, F.; Jiang, X.; Xu, J.; Zhao, D.; Liu, B.; Pan, S.; Dong, X.; Tan, G.; Wei, Z.; et al. Inhibition of Akt Reverses the Acquired Resistance to Sorafenib by Switching Protective Autophagy to Autophagic Cell Death in Hepatocellular Carcinoma. Mol. Cancer Ther. 2014, 13, 1589-1598. [CrossRef]

5. Wilhelm, S.M.; Carter, C.; Tang, L.; Wilkie, D.; McNabola, A.; Rong, H.; Chen, C.; Zhang, X.; Vincent, P.; McHugh, M.; et al. BAY 43-9006 Exhibits Broad Spectrum Oral Antitumor Activity and Targets the RAF/MEK/ERK Pathway and Receptor Tyrosine Kinases Involved in Tumor Progression and Angiogenesis. Cancer Res. 2004, 64, 7099-7109. [CrossRef]

6. Ciamporcero, E.; Miles, K.M.; Adelaiye, R.; Ramakrishnan, S.; Shengyu, K.; Ku, S.Y.; Pizzimenti, S.; Sennino, B.; Barrera, G.; Pili, R. Combination Strategy Targeting VEGF and HGF/c-met in Human Renal Cell Carcinoma Models. Mol. Cancer Ther. 2015, 14, 101-110. [CrossRef]

7. Van Malenstein, H.; Dekervel, J.; Verslype, C.; Van Cutsem, E.; Windmolders, P.; Nevens, F.; Van Pelt, J. Long-term exposure to sorafenib of liver cancer cells induces resistance with epithelial-to-mesenchymal transition, increased invasion and risk of rebound growth. Cancer Lett. 2013, 329, 74-83. [CrossRef]

8. Villanueva, A.; Llovet, J.M. Second-Line Therapies in Hepatocellular Carcinoma: Emergence of Resistance to Sorafenib: Figure 1. Clin. Cancer Res. 2012, 18, 1824-1826. [CrossRef]

9. Farazi, P.A.; Depinho, R.A. Hepatocellular carcinoma pathogenesis: From genes to environment. Nat. Rev. Cancer 2006, 6, 674-687. [CrossRef]

10. Kharaziha, P.; Chioureas, D.; Baltatzis, G.; Fonseca, P.; Rodriguez, P.; Gogvadze, V.; Lennartsson, L.; Björklund, A.-C.; Zhivotovsky, B.; Grandér, D.; et al. Sorafenib-induced defective autophagy promotes cell death by necroptosis. Oncotarget 2015, 6, 37066-37082. [CrossRef]

11. Ramírez-Labrada, A.; López-Royuela, N.; Jarauta, V.; Galán-Malo, P.; Azaceta, G.; Palomera, L.; Pardo, J.; Anel, A.; Marzo, I.; Naval, J. Two death pathways induced by sorafenib in myeloma cells: Puma-mediated apoptosis and necroptosis. Clin. Transl. Oncol. 2014, 17, 121-132. [CrossRef] [PubMed]

12. Locatelli, S.L.; Cleris, L.; Stirparo, G.G.; Tartari, S.; Saba, E.; Pierdominici, M.; Malorni, W.; Carbone, A.; Anichini, A.; Carlo-Stella, C. BIM upregulation and ROS-dependent necroptosis mediate the antitumor effects of the HDACi Givinostat and Sorafenib in Hodgkin lymphoma cell line xenografts. Leukemia 2014, 28, 1861-1871. [CrossRef] [PubMed]

13. Martens, S.; Jeong, M.; Tonnus, W.; Feldmann, F.; Hofmans, S.; Goossens, V.; Takahashi, N.; Braesen, J.H.; Lee, E.-W.; Van Der Veken, P.; et al. Sorafenib tosylate inhibits directly necrosome complex formation and protects in mouse models of inflammation and tissue injury. Cell Death Dis. 2017, 8, e2904. [CrossRef] [PubMed]

14. Sun, L.; Wang, H.; Wang, Z.; He, S.; Chen, S.; Liao, D.; Wang, L.; Yan, J.; Liu, W.; Lei, X.; et al. Mixed Lineage Kinase Domain-like Protein Mediates Necrosis Signaling Downstream of RIP3 Kinase. Cell 2012, 148, 213-227. [CrossRef] [PubMed]

15. Ito, Y.; Ofengeim, D.; Najafov, A.; Das, S.; Saberi, S.; Li, Y.; Hitomi, J.; Zhu, H.; Chen, H.; Mayo, L.; et al. RIPK1 mediates axonal degeneration by promoting inflammation and necroptosis in ALS. Science 2016, 353, 603-608. [CrossRef]

16. Liang, Y.; Zheng, T.; Song, R.; Wang, J.; Yin, D.; Wang, L.; Liu, H.; Tian, L.; Fang, X.; Meng, X.; et al. Hypoxia-mediated sorafenib resistance can be overcome by EF24 through Von Hippel-Lindau tumor suppressor-dependent HIF- $1 \alpha$ inhibition in hepatocellular carcinoma. Hepatology 2013, 57, 1847-1857. [CrossRef]

17. Unruh, A.; Ressel, A.; Mohamed, H.G.; Johnson, R.S.; Nadrowitz, R.; Richter, E.; Katschinski, D.M.; Wenger, R.H. The hypoxiainducible factor- $1 \alpha$ is a negative factor for tumor therapy. Oncogene 2003, 22, 3213-3220. [CrossRef]

18. Xie, G.; Liu, Y.; Yao, Q.; Zheng, R.; Zhang, L.; Lin, J.; Guo, Z.; Du, S.; Ren, C.; Yuan, Q.; et al. Hypoxia-induced angiotensin II by the lactate-chymase-dependent mechanism mediates radioresistance of hypoxic tumor cells. Sci. Rep. 2017, 7, 42396. [CrossRef]

19. Hartl, F.U.; Bracher, A.; Hayer-Hartl, M. Molecular chaperones in protein folding and proteostasis. Nat. Cell Biol. 2011, 475, 324-332. [CrossRef]

20. Verba, K.A.; Wang, R.Y.-R.; Arakawa, A.; Liu, Y.; Shirouzu, M.; Yokoyama, S.; Agard, D. Atomic structure of Hsp90-Cdc37-Cdk4 reveals that Hsp90 traps and stabilizes an unfolded kinase. Science 2016, 352, 1542-1547. [CrossRef]

21. Li, W.; Li, Y.; Guan, S.; Fan, J.; Cheng, C.; Bright, A.; Chin, C.; Chen, M.; Woodley, D. Extracellular heat shock protein-90alpha: Linking hypoxia to skin cell motility and wound healing. J. Invest. Dermatol. 2007, 127, S36.

22. Fike, C.D.; Pfister, S.L.; Slaughter, J.C.; Kaplowitz, M.R.; Zhang, Y.; Zeng, H.; Frye, N.R.; Aschner, J.L. Protein complex formation with heat shock protein 90 in chronic hypoxia-induced pulmonary hypertension in newborn piglets. Am. J. Physiol. Circ. Physiol. 2010, 299, H1190-H1204. [CrossRef] [PubMed]

23. Rohwer, N.; Cramer, T. Hypoxia-mediated drug resistance: Novel insights on the functional interaction of HIFs and cell death pathways. Drug Resist. Updates 2011, 14, 191-201. [CrossRef] [PubMed] 
24. Lu, X.; Xiao, L.; Wang, L.; Ruden, D.M. Hsp90 inhibitors and drug resistance in cancer: The potential benefits of combination therapies of Hsp90 inhibitors and other anti-cancer drugs. Biochem. Pharmacol. 2012, 83, 995-1004. [CrossRef] [PubMed]

25. Zhang, J.; Li, H.; Huang, Z.; He, Y.; Zhou, X.; Huang, T.; Dai, P.; Duan, D.; Ma, X.; Yin, Q.; et al. Hypoxia attenuates Hsp90 inhibitor 17-DMAG-induced cyclin B1 accumulation in hepatocellular carcinoma cells. Cell Stress Chaperones 2016, 21, 339-348. [CrossRef]

26. Pelicano, H.; Carew, J.S.; McQueen, T.J.; Andreeff, M.; Plunkett, W.; Keating, M.J.; Huang, P. Targeting Hsp90 by 17-AAG in leukemia cells: Mechanisms for synergistic and antagonistic drug combinations with arsenic trioxide and Ara-C. Leukemia 2006, 20, 610-619. [CrossRef]

27. Neckers, L. Heat shock protein 90 inhibition by 17-allylamino-17-demethoxygeldanamycin: A novel therapeutic approach for treating hormone-refractory prostate cancer. Clin. Cancer Res. 2002, 8, 962-966.

28. Kim, S.H.; Kang, J.G.; Kim, C.S.; Ihm, S.-H.; Choi, M.G.; Yoo, H.J.; Lee, S.J. The effect of 17-allylamino-17-demethoxygeldanamycin alone or in combination with paclitaxel on anaplastic thyroid carcinoma cells. Endocrine 2014, 48, 886-893. [CrossRef]

29. Mohammadian, M.; Zeynali-Moghaddam, S.; Ansari, M.H.K.; Rami, Y.; Azarbayjani, A.F.; Kheradmand, F. Dihydropyrimidine Dehydrogenase Levels in Colorectal Cancer Cells Treated with a Combination of Heat Shock Protein 90 Inhibitor and Oxaliplatin or Capecitabine. Adv. Pharm. Bull. 2019, 9, 439-444. [CrossRef]

30. Kheradmand, F.; Mohammadian, M.; Zeynali, S.; Azarbaijani, A.F.; Ansari, M.H.K. Cytotoxic effects of the newly-developed chemotherapeutic agents 17-AAG in combination with oxaliplatin and capecitabine in colorectal cancer cell lines. Res. Pharm. Sci. 2017, 12, 517-525. [CrossRef]

31. Yang, Q.; Wang, R.L.; Zhu, L. Chaperone-Mediated Autophagy. Adv. Exp. Med. Biol. 2019, 1206, 435-452. [CrossRef] [PubMed]

32. Ho, P.W.-L.; Leung, C.-T.; Liu, H.; Pang, S.Y.-Y.; Lam, C.S.-C.; Xian, J.; Li, L.; Kung, M.H.-W.; Ramsden, D.B.; Ho, S.-L. Agedependent accumulation of oligomeric SNCA / $\alpha$-synuclein from impaired degradation in mutant LRRK2 knockin mouse model of Parkinson disease: Role for therapeutic activation of chaperone-mediated autophagy (CMA). Autophagy 2020, 16, 347-370. [CrossRef] [PubMed]

33. Hao, Y.; Kacal, M.; Ouchida, A.T.; Zhang, B.; Norberg, E.; Vakifahmetoglu-Norberg, H. Targetome analysis of chaperone-mediated autophagy in cancer cells. Autophagy 2019, 15, 1558-1571. [CrossRef]

34. Dash, S.; Aydin, Y.; Moroz, K. Chaperone-Mediated Autophagy in the Liver: Good or Bad? Cells 2019, 8, 1308. [CrossRef] [PubMed]

35. Ma, L.; Zefu, L.; Liu, Z.; Li, M.; Sui, D.; Liu, Y.; Shao, W.; Wang, B.; Liu, P.; Li, G. 17AAG improves histological and functional outcomes in a rat CCI model through autophagy activation and apoptosis attenuation. Neurosci. Lett. 2015, 599, 1-6. [CrossRef] [PubMed]

36. Chen, P.-H.; Chang, J.T.; Li, L.-A.; Tsai, H.-T.; Shen, M.-Y.; Lin, P. Aryl Hydrocarbon Receptor is a Target of 17-Allylamino-17demethoxygeldanamycin and Enhances its Anticancer Activity in Lung Adenocarcinoma Cells. Mol. Pharmacol. 2012 , 83, 605-612. [CrossRef] [PubMed] 